\title{
Unmixedness of some weighted oriented graphs
}

\author{
Lourdes Cruz*, Yuriko Pitones ${ }^{\dagger}$, and Enrique Reyes ${ }^{\ddagger}$ \\ Departamento de Matemáticas
Centro de Investigación y de Estudios Avanzados del Instituto Politécnico Nacional \\ Apartado Postal 14-740, Ciudad de México \\ 07000 México
}

\begin{abstract}
Let $D=(G, \mathcal{O}, w)$ be a weighted oriented graph whose edge ideal is $I(D)$. In this paper, we characterize the unmixed property of $I(D)$ for each one of the following cases: $G$ is an $S C Q$ graph; $G$ is a chordal graph; $G$ is a simplicial graph; $G$ is a perfect graph; $G$ has no 4- or 5-cycles; $G$ is a graph without 3 - and 5-cycles; and $\operatorname{girth}(G) \geqslant 5$.
\end{abstract}

Keywords: Weighted oriented graphs, edge ideal, unmixed ideal, $S C Q$ graph, perfect graph.

\section{Introduction}

A weighted oriented graph $D$ is a triplet $(G, \mathcal{O}, w)$, where $G$ is a simple graph whose vertex set is $V(G) ; \mathcal{O}$ is an edge orientation of $G$ (an assignment of a direction to each edge of $G$ ); and $w$ is a function $w: V(G) \rightarrow \mathbb{N}$. In this case, $G$ is called the underlying graph of $D$. The vertex set of $D$ is $V(D):=V(G)$, the edge set of $D$, denoted by $E(D)$, is the set of oriented edges of the oriented graph $(G, \mathcal{O})$. The weight of $x \in V(D)$ is $w(x)$ and we denote the set $\{x \in V(D) \mid w(x)>1\}$ by $V^{+}$. If $R=K\left[x_{1}, \ldots, x_{n}\right]$ is a polynomial ring over a field $K$, then the edge ideal of $D$ is $I(D)=\left(x_{i} x_{j}^{w\left(x_{j}\right)} \mid\left(x_{i}, x_{j}\right) \in E(D)\right)$ where $V(D)=\left\{x_{1}, \ldots, x_{n}\right\}$. These ideals (introduced in [10) generalize the usual edge ideals of graphs (see [14]), since if $w(x)=1$ for each $x \in V(D)$, then $I(D)$ is the edge ideal of $G$, i.e. $I(D)=I(G)$. An interest in $I(D)$ comes from coding theory in some studies of Reed-Muller typed codes, (see [1, 9]). Furthermore, some algebraic and combinatorial invariants and properties of $I(D)$ have been studied in some papers $[7,8,10,11$, 15. In particular, in [10] is given a characterization of the irredundant irreducible decomposition of $I(D)$. This characterization permits studying when $I(D)$ is unmixed, using the strong vertex covers (Definition 2.10 and Theorem 2.12). The unmixed property of $I(D)$ have been studied when $G$ is one of the following graphs: cycles in 10; graphs with whiskers in 17, 10]; bipartite graphs in [8, 10; ;raphs without 3- 5- and 7-cycles and König graphs in [11.

In this paper, we study the unmixed property of $I(D)$ for some families of weighted oriented graphs. In Section 2, we give the known results and definitions that we will use in the following sections. In Section 3 (in Theorem 3.10), we characterize when a subset of $V(D)$ is contained in a strong vertex cover. Using this result, we characterize the unmixed property of $I(D)$, when $G$ is a perfect graph (see Theorem 3.11). In Section 4 (in Theorem 4.8), we characterize the unmixed property of $I(D)$ when $G$ is an $S C Q$ graph (see Definition 2.27). These graphs generalize the graph defined in [13] and in the context of this paper, they are important because if $G$ is well-covered such that $G$ is simplicial, $G$ is chordal or $G$ has no some small cycles, then $G$ is an $S C Q$ graph (see

\footnotetext{
*lcruzg@math.cinvestav.mx

$\dagger$ ypitones@math.cinvestav.mx

$\ddagger$ ereyes@math.cinvestav.mx
} 
Remark 2.29 and Theorem 2.30). In [3], using the $S C Q$ graphs the authors characterize the vertex decomposable property of $G$ when each 5 -cycle of $G$ has at least 4 chords. Also, in Section 4, we characterize the unmixed property of $I(D)$ when $G$ is Köning, $G$ is simplicial or $G$ is chordal (see Corollaries 4.2 and 4.9). In Section 5, we characterize the unmixed property of $I(D)$, when $G$ has no 3- or 5-cycles; or $G$ has no 4- or 5-cycles; or $G$ has girth greater than 4 (see Theorems 5.4, 5.10 and 5.13). Finally, in Section 6, we give some examples. Our results generalize the results about the unmixed property of $I(D)$ given in [7, 8, 10, 11, since if $G$ is well-covered and $G$ is one of the following graphs: cycles, graphs with whiskers, bipartite graphs, Köning graphs, or graphs without 3-, 5and 7-cycles, then $G$ is an $S C Q$ graph.

\section{Preliminaries}

In this Section, we give some definitions and well-known results that we will use in the following sections. Let $D=(G, \mathcal{O}, w)$ be a weighted oriented graph, recall that $V^{+}=\{x \in V(D) \mid w(x)>1\}$ and $I(D)=\left(x_{i} x_{j}^{w\left(x_{j}\right)} \mid\left(x_{i}, x_{j}\right) \in E(D)\right)$.

Definition 2.1 Let $x$ be a vertex of $D$, the sets

$$
N_{D}^{+}(x):=\{y \mid(x, y) \in E(D)\} \quad \text { and } \quad N_{D}^{-}(x):=\{y \mid(y, x) \in E(D)\}
$$

are called the out-neighbourhood and the in-neighbourhood of $x$, respectively. The neighbourhood of $x$ is the set $N_{D}(x):=N_{D}^{+}(x) \cup N_{D}^{-}(x)$. Furthermore, $N_{D}[x]:=N_{D}(x) \cup\{x\}$. Also, if $A \subseteq V(D)$ then $N_{D}(A):=\left\{b \in V(D) \mid b \in N_{D}(a)\right.$ for some $\left.a \in A\right\}$.

Definition 2.2 Let $x$ be a vertex of $D$. If $N_{D}^{+}(x)=\emptyset$, then $x$ is called a sink. On the other hand, $x$ is a source if $N_{D}^{-}(x)=\emptyset$.

Remark 2.3 Consider the weighted oriented graph $\tilde{D}=(G, \mathcal{O}, \tilde{w})$ with $\tilde{w}(x)=1$ if $x$ is a source and $\tilde{w}(x)=w(x)$ if $x$ is not a source. Hence, $I(\tilde{D})=I(D)$. Therefore, in this paper, we assume that if $x$ is a source, then $w(x)=1$.

Definition 2.4 The degree of $x \in V(D)$ is $\operatorname{deg}_{G}(x):=\left|N_{D}(x)\right|$ and $N_{G}(x):=N_{D}(x)$.

Definition 2.5 A vertex cover $\mathcal{C}$ of $D$ (resp. of $G$ ) is a subset of $V(D)$ (resp. of $V(G)$ ), such that if $(x, y) \in E(D)$ (resp. $\{x, y\} \in E(G)$ ), then $x \in \mathcal{C}$ or $y \in \mathcal{C}$. A vertex cover $\mathcal{C}$ of $D$ is minimal if each proper subset of $\mathcal{C}$ is not a vertex cover of $D$.

Remark 2.6 Let $\mathcal{C}$ be a vertex cover of $D$ and $e \in E(G)$. Then, $\mathcal{C} \cap e \neq \emptyset$. Furthermore, $e \cap(\mathcal{C} \backslash a) \neq \emptyset$ if $a \notin e, b \in N_{D}(a)$ and $e=\{a, b\}$. Hence, $(\mathcal{C} \backslash a) \cup N_{D}(a)$ is a vertex cover of $D$.

Definition 2.7 Let $\mathcal{C}$ be a vertex cover of $D$, we define the following three sets:

- $L_{1}(\mathcal{C}):=\left\{x \in \mathcal{C} \mid N_{D}^{+}(x) \cap \mathcal{C}^{c} \neq \emptyset\right\}$ where $\mathcal{C}^{c}=V(D) \backslash \mathcal{C}$,

- $L_{2}(\mathcal{C}):=\left\{x \in \mathcal{C} \mid x \notin L_{1}(\mathcal{C})\right.$ and $\left.N_{D}^{-}(x) \cap \mathcal{C}^{c} \neq \emptyset\right\}$,

- $L_{3}(\mathcal{C}):=\mathcal{C} \backslash\left(L_{1}(\mathcal{C}) \cup L_{2}(\mathcal{C})\right)$. 
Remark 2.8 If $\mathcal{C}$ is a vertex cover of $G, x \in V(G) \backslash \mathcal{C}$ and $y \in N_{G}(x)$, then $e:=\{x, y\} \in$ $E(G)$ and $e \cap \mathcal{C} \neq \emptyset$. So, $y \in \mathcal{C}$, since $x \notin \mathcal{C}$. Hence, $N_{G}(x) \subseteq \mathcal{C}$.

Remark 2.9 Let $\mathcal{C}$ be a vertex cover of $D$, then $x \in L_{3}(\mathcal{C})$ if and only if $N_{D}[x] \subseteq \mathcal{C}$. Hence, $L_{3}(\mathcal{C})=\emptyset$ if and only if $\mathcal{C}$ is minimal.

Definition 2.10 A vertex cover $\mathcal{C}$ of $D$ is strong if for each $x \in L_{3}(\mathcal{C})$ there is $(y, x) \in$ $E(D)$ such that $y \in L_{2}(\mathcal{C}) \cup L_{3}(\mathcal{C})=\mathcal{C} \backslash L_{1}(\mathcal{C})$ with $y \in V^{+}($i.e. $w(y)>1$ ).

Definition 2.11 An ideal $I$ of a ring $R$ is unmixed if each one of its associated primes has the same height.

Theorem 2.12 [10, Theorem 31] The following conditions are equivalent:

(1) $I(D)$ is unmixed.

(2) Each strong vertex cover of $D$ has the same cardinality.

(3) $I(G)$ is unmixed and $L_{3}(\mathcal{C})=\emptyset$ for each strong vertex cover $\mathcal{C}$ of $D$.

Definition 2.13 The cover number of $G$ is $\tau(G):=\min \{|\mathcal{C}| \mid \mathcal{C}$ is a vertex cover of $G\}$. Furthermore, a $\tau$-reduction of $G$ is a collection of pairwise disjoint induced subgraphs $H_{1}, \ldots, H_{s}$ of $G$ such that $V(G)=\cup_{i=1}^{s} V\left(H_{i}\right)$ and $\tau(G)=\sum_{i=1}^{s} \tau\left(H_{i}\right)$.

Remark 2.14 We have $\tau(G)=\left|\mathcal{C}_{1}\right|$, for some vertex cover $\mathcal{C}_{1}$. So, $\mathcal{C}_{1}$ is minimal. Thus, by Remark 2.9, $L_{3}\left(\mathcal{C}_{1}\right)=\emptyset$. Hence, $\mathcal{C}_{1}$ is strong. Now, if $I(D)$ is unmixed, then by $(2)$ in Theorem 2.12 $|\mathcal{C}|=\left|\mathcal{C}_{1}\right|=\tau(G)$ for each strong vertex cover $\mathcal{C}$ of $D$.

Definition 2.15 A stable set of $G$ is a subset of $V(G)$ containing no edge of $G$. The stable number of $G$, denoted by $\beta(G)$, is $\beta(G):=\max \{|S| \mid S$ is a stable set of $G\}$. Furthermore $G$ is well-covered if $|S|=\beta(G)$ for each maximal stable set $S$ of $G$.

Remark 2.16 $S$ is a stable set of $G$ if and only if $V(G) \backslash S$ is a vertex cover. Hence, $\tau(G)=|V(G)|-\beta(G)$.

Remark 2.17 [11, Remark 2.12] $G$ is well-covered if and only if $I(G)$ is unmixed.

Definition 2.18 A collection of pairwise disjoint edges of $G$ is called a matching. A perfect matching is a matching whose union is $V(G)$. On the other hand, $G$ is a König graph if $\tau(G)=\nu(G)$ where $\nu(G)$ is the maximum cardinality of a matching of $G$.

Definition 2.19 Let $e$ be an edge of $G$. If $\left\{a, a^{\prime}\right\} \in E(G)$ for each pair of edges, $\{a, b\}$, $\left\{a^{\prime}, b^{\prime}\right\} \in E(G)$ and $e=\left\{b, b^{\prime}\right\}$, then we say that $e$ has the property $(\mathbf{P})$. On the other hand, we say that a matching $P$ of $G$ has the property $(\mathbf{P})$ if each edge of $P$ has the property $(\mathbf{P})$.

Theorem 2.20 [2, Proposition 15] If $G$ is a Köning graph without isolated vertices, then $G$ is well-covered if and only if $G$ has a perfect matching with the property $(\mathbf{P})$.

Definition 2.21 $\mathcal{P}=\left(x_{1}, \ldots, x_{n}\right)$ is a walk (resp. an oriented walk) if $\left\{x_{i}, x_{i+1}\right\} \in E(G)$ for $i=1, \ldots, n-1$. In this case, $\mathcal{P}$ is a path (resp. an oriented path) if $x_{1}, \ldots, x_{n}$ are different. On the other hand, a walk (resp. an oriented walk), $C=\left(z_{1}, z_{2}, \ldots, z_{n}, z_{1}\right)$ is a $n$-cycle (resp. an oriented $n$-cycle) if $\left(z_{1}, \ldots, z_{n}\right)$ is a path (resp. is an oriented path). 
Definition 2.22 Let $A$ be a subset of $V(G)$, then the graph induced by $A$, denoted by $G[A]$, is the subgraph $G_{1}$ of $G$ with $V\left(G_{1}\right)=A$ and $E\left(G_{1}\right)=\{e \in E(G) \mid e \subseteq A\}$. On the other hand, a subgraph $H$ of $G$ is induced if there is $B \subseteq V(G)$ such that $H=G[B]$. A cycle $C$ of $G$ is induced if $C$ is an induced subgraph of $G$.

Definition 2.23 A weighted oriented graph $D^{\prime}=\left(G^{\prime}, \mathcal{O}^{\prime}, w^{\prime}\right)$ is a weighted oriented subgraph of $D=(G, \mathcal{O}, w)$, if $\left(G^{\prime}, \mathcal{O}^{\prime}\right)$ is an oriented subgraph of $(G, \mathcal{O})$ and $w^{\prime}(x)=w(x)$ for each $x \in V\left(G^{\prime}\right)$. Furthermore, $D^{\prime}$ is an induced weighted oriented subgraph of $D$ if $G^{\prime}$ is an induced subgraph of $G$.

Definition 2.24 A vertex $v$ is called simplicial if the induced subgraph $H=G\left[N_{G}[v]\right]$ is a complete graph with $k=|V(H)|-1$, in this case, $H$ is called $k$-simplex (or simplex). The set of simplexes of $G$ is denoted by $S_{G}$. $G$ is a simplicial graph if every vertex of $G$ is a simplicial vertex of $G$ or is adjacent to a simplicial vertex of $G$.

Definition 2.25 The minimum length of a cycle (contained) in a graph $G$, is called the girth of $G$. On the other hand, $G$ is a chordal graph if the induced cycles are 3-cycles.

Theorem 2.26 [12, Theorems 1 and 2] If $G$ is a chordal or simplicial graph, then $G$ is well-covered if and only if every vertex of $G$ belongs to exactly one simplex of $G$.

Definition 2.27 An induced 5-cycle $C$ of $G$ is called basic if $C$ does not contain two adjacent vertices of degree three or more in $G$. $G$ is an $S C Q$ graph (or $G \in S C Q$ ) if $G$ satisfies the following conditions:

(i) There is $Q_{G}$ such that $Q_{G}=\emptyset$ or $Q_{G}$ is a matching of $G$ with the property (P).

(ii) $\left\{V(H) \mid H \in S_{G} \cup C_{G} \cup Q_{G}\right\}$ is a partition of $V(G)$, where $C_{G}$ is the set of basic 5 -cycles.

In the following three results, we use the graphs of Figure 1 .

Theorem 2.28 [6, Theorem 1.1] If $G$ is connected without 4- and 5-cycles, then $G$ is well-covered if and only if $G \in\left\{C_{7}, T_{10}\right\}$ or $\left\{V(H) \mid H \in S_{G}\right\}$ is a partition of $V(G)$.

Remark 2.29 Suppose $G$ is well-covered. If $G$ is simplicial, or $G$ is chordal or $G$ is a graph without 4 - and 5 -cycles and $G \notin\left\{C_{7}, T_{10}\right\}$. Then, by Theorems 2.26 and 2.28. $\left\{V(H) \mid H \in S_{G}\right\}$ is a partition of $V(G)$. Therefore, $G$ is an $S C Q$ graph with $C_{G}=Q_{G}=\emptyset$.

Theorem 2.30 [5, Theorem 2 and Theorem 3] If $G$ is a connected graph without 3and 4-cycles, then $G$ is well-covered if and only if $G \in\left\{K_{1}, C_{7}, P_{10}, P_{13}, P_{14}, Q_{13}\right\}$ or $\left\{V(H) \mid H \in S_{G} \cup C_{G}\right\}$ is a partition of $V(G)$.

Definition 2.31 The complement of $G$, denoted by $\bar{G}$, is the graph with $V(\bar{G})=V(G)$ such that for each pair of different vertices $x$ and $y$ of $D$, we have that $\{x, y\} \in E(\bar{G})$ if and only if $\{x, y\} \notin E(G)$.

Definition 2.32 A $k$-colouring of $G$ is a function $c: V(G) \rightarrow\{1,2, \ldots, k\}$ such that $c(u) \neq c(v)$ if $\{u, v\} \notin E(G)$. The smallest integer $k$ such that $G$ has a $k$-colouring is called the chromatic number of $G$ and it is denoted by $\chi(G)$. On the other hand, the clique number, denoted by $\omega(G)$ is the size of the largest complete subgraph of $G$. Finally, $G$ is perfect if $\chi(H)=\omega(H)$ for every induced subgraph $H$ of $G$. 

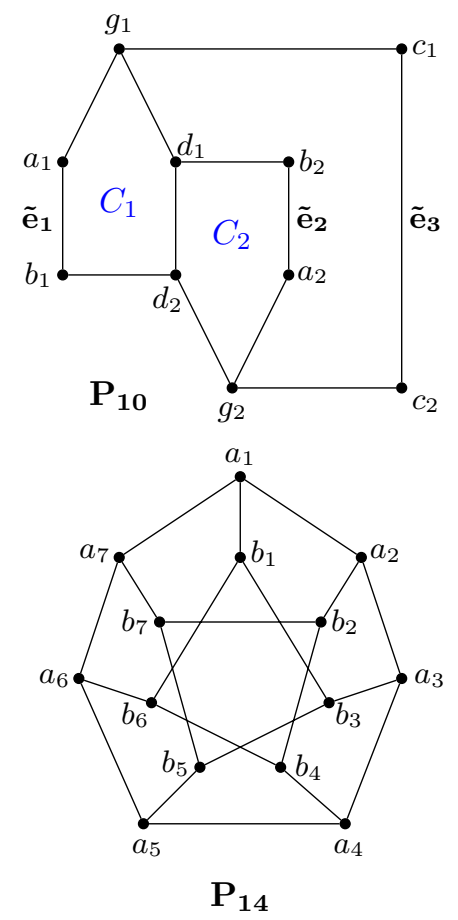
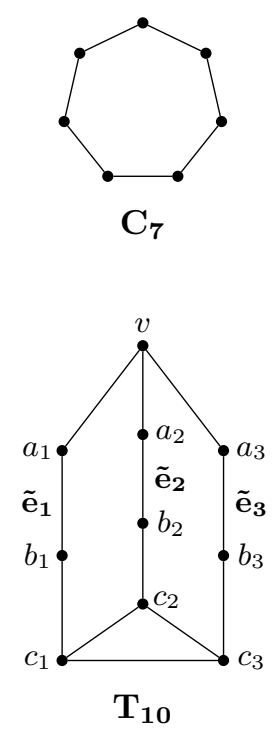

Figure 1
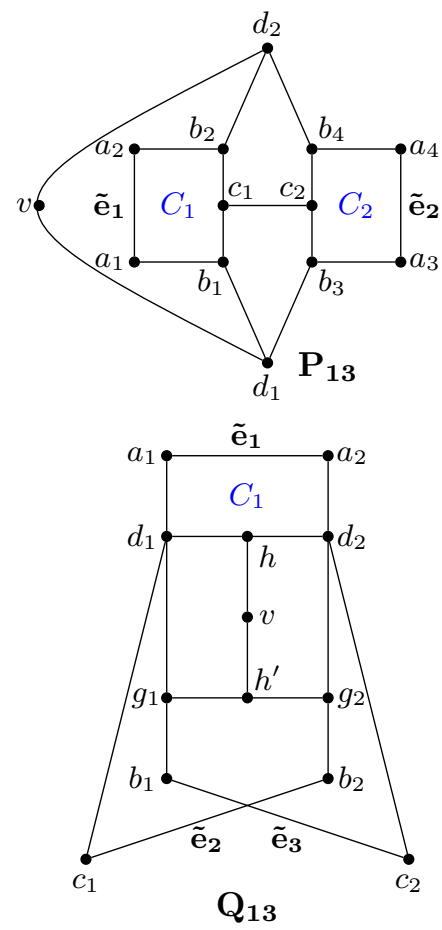

is a complete subgraph of $\bar{G}$. Hence, $\beta(G)=\omega(\bar{G})$.

Theorem 2.34 [4, Theorem 5.5.3] $G$ is perfect if and only if $\bar{G}$ is perfect.

\section{Strong vertex cover and $\star$-semi-forest}

Let $D=(G, \mathcal{O}, w)$ be a weighted oriented graph. In this Section, we introduce the unicycle oriented subgraphs (Definition 3.2), the root oriented trees (Definition [3.3), and the $\star$-semi-forests of $D$ (Definition 3.4). With this definitions, we characterize when a subset of $V(G)$ is contained in a strong vertex cover (see Theorem 3.10). Using this result, we characterize when $I(D)$ is unmixed if $G$ is a perfect graph (see Definition 2.32 and Theorem 3.11).

Proposition 3.1 If $\mathcal{C}$ is a vertex cover of $D$ such that $N_{D}^{+}(A) \subseteq \mathcal{C}$ and $A \subseteq V^{+}$, then there is a strong vertex cover $\mathcal{C}^{\prime}$ of $D$, such that $N_{D}^{+}(A) \subseteq \mathcal{C}^{\prime} \subseteq \mathcal{C}$.

Proof. First, we prove that there is a vertex cover $\mathcal{C}^{\prime}$ such that $L_{3}\left(\mathcal{C}^{\prime}\right) \subseteq N_{D}^{+}(A) \subseteq \mathcal{C}^{\prime} \subseteq \mathcal{C}$. We take $L:=N_{D}^{+}(A)$. If $L_{3}(\mathcal{C}) \subseteq L$, then we take $\mathcal{C}^{\prime}=\mathcal{C}$. Now, we suppose there is $a_{1} \in L_{3}(\mathcal{C}) \backslash L$, then by Remark 2.9, $N_{D}\left[a_{1}\right] \subseteq \mathcal{C}$. Thus, $\mathcal{C}_{1}=\mathcal{C} \backslash\left\{a_{1}\right\}$ is a vertex cover and $L \subseteq \mathcal{C}_{1}$, since $L \subseteq \mathcal{C}$ and $a_{1} \notin L$. Now, we suppose that there are vertex 
covers $\mathcal{C}_{0}, \ldots, \mathcal{C}_{k}$, such that $L \subseteq \mathcal{C}_{i}=\mathcal{C}_{i-1} \backslash\left\{a_{i}\right\}$ and $a_{i} \in L_{3}\left(\mathcal{C}_{i-1}\right) \backslash L$ for $i=1, \ldots, k$ where $\mathcal{C}_{0}=\mathcal{C}$ and we give the following recursively process: If $L_{3}\left(\mathcal{C}_{k}\right) \subseteq L$, then we take $\mathcal{C}^{\prime}=\mathcal{C}_{k}$. Now, if there is $a_{k+1} \in L_{3}\left(\mathcal{C}_{k}\right) \backslash L$, then by Remark 2.9] $N_{D}\left[a_{k+1}\right] \subseteq \mathcal{C}_{k}$. Consequently, $\mathcal{C}_{k+1}:=\mathcal{C}_{k} \backslash\left\{a_{k+1}\right\}$ is a vertex cover. Also, $L \subseteq \mathcal{C}_{k+1}$, since $L \subseteq \mathcal{C}_{k}$ and $a_{k+1} \notin L$. This process is finite, since $|V(D)|$ is finite. Hence, there is $m$ such that $L_{3}\left(\mathcal{C}_{m}\right) \subseteq L \subseteq \mathcal{C}_{m} \subseteq \mathcal{C}$. Therefore, we take $\mathcal{C}^{\prime}=\mathcal{C}_{m}$.

Now, we prove that $\mathcal{C}^{\prime}$ is strong. We take $x \in L_{3}\left(\mathcal{C}^{\prime}\right)$, then $x \in L=N_{D}^{+}(A)$, since $L_{3}\left(\mathcal{C}^{\prime}\right) \subseteq L$. Thus, $(y, x) \in E(D)$ for some $y \in A \subseteq V^{+}$. Hence, $y \in \mathcal{C}^{\prime}$, since $x \in L_{3}\left(\mathcal{C}^{\prime}\right)$. Also, $y \notin L_{1}\left(\mathcal{C}^{\prime}\right)$, since $N_{D}^{+}(y) \subseteq N_{D}^{+}(A) \subseteq \mathcal{C}^{\prime}$. Hence, $y \in\left(\mathcal{C}^{\prime} \backslash L_{1}\left(\mathcal{C}^{\prime}\right)\right) \cap V^{+}$. Therefore, $\mathcal{C}^{\prime}$ is strong.

Definition 3.2 If $B$ is a weighted oriented subgraph of $D$ with exactly one cycle $C$, then $B$ is called unicycle oriented graph when $B$ satisfies the following conditions:

(i) $C$ is an oriented cycle in $B$ and there is an oriented path from $C$ to $y$ in $B$, for each $y \in V(B) \backslash V(C)$.

(ii) If $x \in V(B)$ with $w(x)=1$, then $\operatorname{deg}_{B}(x)=1$.

Definition 3.3 A weighted oriented subgraph $T$ of $D$ without cycles, is a root oriented tree $(R O T)$ with parent $v \in V(T)$ when $T$ satisfies the following properties:

(i) If $x \in V(T) \backslash\{v\}$, there is an oriented path $\mathcal{P}$ in $T$ from $v$ to $x$.

(ii) If $x \in V(T)$ with $w(x)=1$, then $\operatorname{deg}_{T}(x)=1$ and $x \neq v$ or $V(T)=\{v\}$ and $x=v$.

Definition 3.4 A weighted oriented subgraph $H$ of $D$ is a $\star$-semi-forest if there are root oriented trees $T_{1}, \ldots, T_{r}$ whose parents are $v_{1}, \ldots, v_{r}$ and unicycle oriented subgraphs $B_{1}, \ldots, B_{s}$ such that $H=\left(\cup_{i=1}^{r} T_{i}\right) \cup\left(\cup_{j=1}^{s} B_{j}\right)$ with the following conditions:

(i) $V\left(T_{1}\right), \ldots, V\left(T_{r}\right), V\left(B_{1}\right), \ldots, V\left(B_{s}\right)$ is a partition of $V(H)$.

(ii) There is $W=\left\{w_{1}, \ldots, w_{r}\right\} \subseteq V(D) \backslash V(H)$ such that $w_{i} \in N_{D}\left(v_{i}\right)$ for $i=1, \ldots, r$ (it is possible that $w_{i}=w_{j}$ for some $1 \leqslant i<j \leqslant r$ ).

(iii) There is a partition $W_{1}, W_{2}$ of $W$ such that $W_{1}$ is a stable set of $D, W_{2} \subseteq V^{+}$and $\left(w_{i}, v_{i}\right) \in E(D)$ if $w_{i} \in W_{2}$. Also, $N_{D}^{+}\left(W_{2} \cup \tilde{H}\right) \cap W_{1}=\emptyset$, where

$$
\tilde{H}=\left\{x \in V(H) \mid \operatorname{deg}_{H}(x) \geqslant 2\right\} \cup\left\{v_{i} \mid \operatorname{deg}_{H}\left(v_{i}\right)=1\right\} .
$$

Remark 3.5 By Definition 3.2 and Definition 3.3 , we have $\tilde{H} \subseteq V^{+}$. Furthermore, if $v_{i}$ is a parent vertex of $T_{i}$, with $\operatorname{deg}_{H}\left(v_{i}\right) \geqslant 1$, then $v_{i} \in \tilde{H}$.

Lemma 3.6 If $H$ is a «-semi-forest of $D$, then

$$
V(H) \subseteq N_{D}\left(W_{1}\right) \cup N_{D}^{+}\left(W_{2} \cup \tilde{H}\right) .
$$

Proof. We take $x \in V(H)$. Since $H=\left(\cup_{i=1}^{r} T_{i}\right) \cup\left(\cup_{j=1}^{s} B_{j}\right)$, we have two cases:

Case 1) $x \in V\left(B_{j}\right)$ for some $1 \leqslant j \leqslant s$. Let $C$ be the oriented cycle of $B_{j}$. If $x \in V(C)$, then there is $y_{1} \in V(C)$ such that $\left(y_{1}, x\right) \in E(C)$. Furthermore, $\operatorname{deg}_{H}\left(y_{1}\right) \geqslant \operatorname{deg}_{C}\left(y_{1}\right)=$ 2 , then $y_{1} \in \tilde{H}$. Hence, $x \in N_{D}^{+}\left(y_{1}\right) \subseteq N_{D}^{+}(\tilde{H})$. Now, if $x \in V\left(B_{j}\right) \backslash V(C)$, then there is an oriented path $\mathcal{P}$ in $B_{j}$ from $C$ to $x$. Thus, there is $y_{2} \in V(\mathcal{P})$ such that $\left(y_{2}, x\right) \in E(\mathcal{P})$. If $|V(\mathcal{P})|>2$, then $\operatorname{deg}_{H}\left(y_{2}\right) \geqslant \operatorname{deg}_{\mathcal{P}}\left(y_{2}\right)=2$. If $|V(\mathcal{P})|=2$, then $y_{2} \in V(C)$ and $\operatorname{deg}_{H}\left(y_{2}\right)>\operatorname{deg}_{C}\left(y_{2}\right)=2$. Therefore, $y_{2} \in \tilde{H}$ and $x \in N_{D}^{+}(\tilde{H})$. 
Case 2) $x \in V\left(T_{i}\right)$ for some $1 \leqslant i \leqslant r$. First, assume $x=v_{i}$, then there is $w_{i} \in W$ such that $x \in N_{D}\left(w_{i}\right)$. Consequently, $x \in N_{D}\left(W_{1}\right)$ if $w_{i} \in W_{1}$ and, by (iii) of Definition 3.4 $x \in N_{D}^{+}\left(w_{i}\right) \subseteq N_{D}^{+}\left(W_{2}\right)$ if $w_{i} \in W_{2}$. Now, we suppose $x \neq v_{i}$, then there is an oriented path $\mathcal{L}$, from $v_{i}$ to $x$. Consequently, there is $y_{3} \in V(\mathcal{L})$ such that $\left(y_{3}, x\right) \in E(D)$. If $y_{3} \neq v_{i}$, then $\operatorname{deg}_{H}\left(y_{3}\right) \geqslant \operatorname{deg}_{\mathcal{L}}\left(y_{3}\right)=2$. Thus, $y_{3} \in \tilde{H}$ and $x \in N_{D}^{+}(\tilde{H})$. Finally, if $y_{3}=v_{i}$, then $\operatorname{deg}_{H}\left(y_{3}\right) \geqslant 1$. Hence, by Remark 3.5, $y_{3} \in \tilde{H}$ and $x \in N_{D}^{+}(\tilde{H})$.

Remark 3.7 Sometimes to stress the relation between $W$ and $H$ in Definition 3.4 $W$ is denoted by $W^{H}$. Similarly, $W_{1}^{H}$ and $W_{2}^{H}$. If $\left\{T_{1}, \ldots, T_{r}\right\}=\emptyset$, then $W^{H}=W_{1}^{H}=$ $W_{2}^{H}=\emptyset$.

Lemma 3.8 Let $K$ be a weighted oriented subgraph of $D$. If $H$ is a maximal ROT in $K$ with parent $v$, or $H$ is a maximal unicycle oriented subgraph in $K$ whose cycle is $C$, then there is no $(y, x) \in E(K)$ with $x \in V(K) \backslash V(H)$ and $y \in V^{+} \cap V(H)$.

Proof. By contradiction suppose there is $(y, x) \in E(K)$ with $x \in V(K) \backslash V(H)$ and $y \in V^{+} \cap V(H)$. Thus, $H \subsetneq H_{1}:=H \cup\{(y, x)\} \subseteq K$. If $H$ is a unicycle oriented subgraph with cycle $C$ (resp. $H$ is a ROT), then there is an oriented path $\mathcal{P}$ from $C$ (resp. from $v$ ) to $y$. Consequently, $\mathcal{P} \cup\{(y, x)\}$ is an oriented path from $C$ (resp. from $v$ ) to $x$ in $H_{1}$. Furthermore, $H_{1}$ has exactly one cycle (resp. has no cycles), since $\operatorname{deg}_{H_{1}}(x)=1$ and $V\left(H_{1}\right) \backslash V(H)=\{x\}$.

Now, we take $z \in V\left(H_{1}\right)$ with $w(z)=1$, then $z=x$ or $z \in V(H)$. We prove $\operatorname{deg}_{H_{1}}(z)=1$. If $z=x$, then $\operatorname{deg}_{H_{1}}(x)=1$. Now, if $z \in V(H)$, then $z \neq y$, since $y \in V^{+}$. So, $\operatorname{deg}_{H_{1}}(z)=\operatorname{deg}_{H}(z)$, since $N_{H_{1}}(x)=\{y\}$. If $H$ is a ROT with $V(H)=\{v\}$, then $y=z=v$. A contradiction, since $w(z)=1$ and $y \in V^{+}$. Consequently, by (ii) in Definitions 3.2 and 3.3. $\operatorname{deg}_{H_{1}}(z)=\operatorname{deg}_{H}(z)=1$. Hence, $H_{1}$ is a unicycle oriented subgraph with cycle $C$ (resp. is a ROT with parent $v$ ) of $K$. This is a contradiction, since $H \subsetneq H_{1} \subseteq K$ and $H$ is maximal.

Definition 3.9 Let $K$ be a weighted oriented subgraph of $D$ and $H$ a $\star$-semi-forest of $D$. We say $H$ is a generating $\star$-semi-forest of $K$ if $V(K)=V(H)$.

Theorem 3.10 Let $K$ be an induced weighted oriented subgraph of D. Hence, the following conditions are equivalent:

(1) There is a strong vertex cover $\mathcal{C}$ of $D$, such that $V(K) \subseteq \mathcal{C}$.

(2) There is a generating $\star$-semi-forest $H$ of $K$.

Proof. (2) $\Rightarrow(1)$ Let $\mathcal{C}_{1}$ be a minimal vertex cover of $D$. By (2), $K$ has a generating $\star$-semi-forest $H$. Now, using the notation of Definition 3.4, we take $\mathcal{C}_{2}=\left(\mathcal{C}_{1} \backslash W_{1}\right) \cup$ $N_{D}\left(W_{1}\right) \cup N_{D}^{+}\left(W_{2} \cup \tilde{H}\right)$. By Remark 2.6 $\mathcal{C}_{2}$ is a vertex cover of $D$. Since $W_{1}$ is a stable set, $N_{D}\left(W_{1}\right) \cap W_{1}=\emptyset$. Then, $\mathcal{C}_{2} \cap W_{1}=\emptyset$, since $N_{D}^{+}\left(W_{2} \cup \tilde{H}\right) \cap W_{1}=\emptyset$. By Remark 3.5 and (iii) in Definition 3.4. $\tilde{H} \cup W_{2} \subseteq V^{+}$. So, by Proposition 3.1, there is a strong vertex cover $\mathcal{C}$ of $D$ such that $N_{D}^{+}\left(W_{2} \cup \tilde{H}\right) \subseteq \mathcal{C} \subseteq \mathcal{C}_{2}$. Consequently, $\mathcal{C} \cap W_{1}=\emptyset$, since $\mathcal{C}_{2} \cap W_{1}=\emptyset$. Thus, $N_{D}\left(W_{1}\right) \subseteq \mathcal{C}$, since $\mathcal{C}$ is a vertex cover. Then, by Lemma 3.6. $V(H) \subseteq N_{D}\left(W_{1}\right) \cup N_{D}^{+}\left(W_{2} \cup \tilde{H}\right) \subseteq \mathcal{C}$. Hence, $V(K) \subseteq \mathcal{C}$, since $H$ is a generating

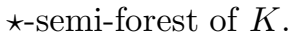

$(1) \Rightarrow(2)$ We have, $\mathcal{C}$ is a strong vertex cover such that $V(K) \subseteq \mathcal{C}$. If $A:=L_{1}(\mathcal{C}) \cap$ $V(K)=\left\{v_{1}, \ldots, v_{s}\right\}$, then there is $w_{i} \in V(D) \backslash \mathcal{C} \subseteq V(D) \backslash V(K)$ such that $\left(v_{i}, w_{i}\right) \in$ 
$E(D)$. We take the ROT's $M_{1}=\left\{v_{1}\right\}, \ldots, M_{s}=\left\{v_{s}\right\}$ and sets $W_{1}^{i}=\left\{w_{i}\right\}$ and $W_{2}^{i}=\emptyset$ for $i=1, \ldots, s$.

Now, we will give a recursive process to obtain a generating $\star$-semi-forest of $K$. For this purpose, suppose we have connected $\star$-semi-forests $M_{s+1}, \ldots, M_{l}$ of $K \backslash A$ with subsets $W_{1}^{s+1}, \ldots, W_{1}^{l}, W_{2}^{s+1}, \ldots, W_{2}^{l} \subseteq V(D) \backslash V(K)$ and $V^{s+1}, \ldots, V^{l} \subseteq V(K)$ such that for each $s<j \leqslant l$, they satisfies the following conditions:

(a) $V^{j}=\left\{v_{j}\right\}$ if $M_{j}$ is a ROT with parent $v_{j}$ or $V^{j}$ is the cycle of $M_{j}$ if $M_{j}$ is a unicycle oriented subgraph,

(b) $M_{j}$ is a maximal ROT in $K^{j}:=K \backslash \cup_{i=1}^{j-1} V\left(M_{i}\right)$ with parent in $V^{j}$ or $M_{j}$ is a maximal unicycle oriented subgraph in $K^{j}$ with cycle $V^{j}$.

(c) $W_{1}^{j} \cap \mathcal{C}=\emptyset$ and $W_{2}^{j} \subseteq\left(\mathcal{C} \backslash\left(L_{1}(\mathcal{C}) \cup V(K)\right)\right) \cap V^{+}$.

Hence, we take $K^{l+1}:=K \backslash\left(\cup_{i=1}^{l} V\left(M_{i}\right)\right)$. This process starts with $l=s$; in this case, $K^{s+1}:=K \backslash\left(\cup_{i=1}^{s} V\left(M_{i}\right)\right)=K \backslash A$; furthermore, if $A=\emptyset$, then $K^{1}=K$. Continuing with the recursive process, if $K^{l+1}=\emptyset$, then $V(K)=\cup_{i=1}^{l} V\left(M_{i}\right)$ and we stop the process. Now, if $K^{l+1} \neq \emptyset$, then we will construct a connected $\star$-semi-forest $M_{l+1}$ of $K^{l+1}$ in the following way:

Case (1) $L_{2}(\mathcal{C}) \cap V\left(K^{l+1}\right) \neq \emptyset$. Then, there is $z \in L_{2}(\mathcal{C}) \cap V\left(K^{l+1}\right)$. Thus, there is $\left(z^{\prime}, z\right) \in E(D)$ with $z^{\prime} \notin \mathcal{C}$. We take a maximal ROT $M_{l+1}$ in $K^{l+1}$, whose parent is $z$. Also, we take $V^{l+1}=\left\{v_{l+1}\right\}=\{z\}, W_{1}^{l+1}=\left\{w_{l+1}\right\}=\left\{z^{\prime}\right\}$ and $W_{2}^{l+1}=\emptyset$. Hence, $M_{l+1}$ satisfies (a), (b) and (c), since $z^{\prime} \notin \mathcal{C}$ and $W_{2}^{l+1}=\emptyset$.

Case (2) $L_{2}(\mathcal{C}) \cap V\left(K^{l+1}\right)=\emptyset$. Then, $V\left(K^{l+1}\right) \subseteq L_{3}(\mathcal{C})$, since $K^{l+1} \subseteq K \backslash A \subseteq \mathcal{C} \backslash L_{1}(\mathcal{C})$. We take $x \in V\left(K^{l+1}\right)$, then there is $x_{1} \in\left(\mathcal{C} \backslash L_{1}(\mathcal{C})\right) \cap V^{+}$such that $\left(x_{1}, x\right) \in E(D)$, since $\mathcal{C}$ is strong. If $x_{1} \in V\left(K^{l+1}\right)$, then there is $x_{2} \in\left(\mathcal{C} \backslash L_{1}(\mathcal{C})\right) \cap V^{+}$such that $\left(x_{2}, x_{1}\right) \in E(D)$, since $\mathcal{C}$ is strong. Continuing with this process we obtain a maximal path $\mathcal{P}=\left(x_{r}, x_{r-1}, \ldots, x_{1}, x\right)$ such that $x_{r-1}, \ldots, x_{1}, x$ are different in $V\left(K^{l+1}\right)$ and $x_{1}, \ldots, x_{r} \in\left(\mathcal{C} \backslash L_{1}(\mathcal{C})\right) \cap V^{+}$. Thus, $x_{r} \notin \cup_{j=1}^{s} V\left(M_{j}\right)$, since $x_{r} \notin L_{1}(\mathcal{C})$. Now, suppose $x_{r} \in V\left(M_{j}\right)$ for some $s<j \leqslant l$. So, $\left(x_{r}, x_{r-1}\right) \in E\left(K^{j}\right), x_{r-1} \in V\left(K^{j}\right) \backslash V\left(M_{j}\right)$ and $x_{r} \in V^{+} \cap V\left(M_{j}\right)$. Furthermore, $N_{D}^{+}\left(x_{r}\right) \cap W_{1}^{j}=\emptyset$, since $x_{r} \in \mathcal{C} \backslash L_{1}(\mathcal{C})$ and $\mathcal{C} \cap W_{1}^{j}=\emptyset$. A contradiction, by Lemma 3.8, since $W^{j} \cap V\left(K^{j}\right)=\emptyset$. Hence, $x_{r} \notin \cup_{j=1}^{l} V\left(M_{j}\right)$. Consequently, $x_{r} \notin V(K)$ or $x_{r} \in V\left(K^{l+1}\right)$.

Case (2.a) $x_{r} \notin V(K)$. Then, take a maximal ROT $M_{l+1}$ in $K^{l+1}$ whose parent is $x_{r-1}$. Also, we take $V^{l+1}=\left\{v_{l+1}\right\}=\left\{x_{r-1}\right\}, W_{1}^{l+1}=\emptyset$; and $W_{2}^{l+1}=\left\{w_{l+1}\right\}=\left\{x_{r}\right\}$. Thus, $M_{l+1}$ satisfies (a), (b) and (c), since $W_{1}^{l+1}=\emptyset, x_{r} \in\left(\mathcal{C} \backslash L_{1}(\mathcal{C})\right) \cap V^{+}$and $x_{r} \notin V(K)$. Case (2.b) $x_{r} \in V\left(K^{l+1}\right)$. Then, $x_{r} \in L_{3}(\mathcal{C})$, since $V\left(K^{l+1}\right) \subseteq L_{3}(\mathcal{C})$. Hence, there is $\left.\overline{x_{r+1} \in(\mathcal{C}} \backslash L_{1}(\mathcal{C})\right) \cap V^{+}$such that $\left(x_{r+1}, x_{r}\right) \in E(D)$, Then $\tilde{\mathcal{P}}=\left(x_{r+1}, x_{r}, \ldots, x_{1}, x\right)$ is an oriented walk. By the maximality of $\mathcal{P}$, we have that $x_{r} \in\left\{x_{r-1}, \ldots, x_{1}, x\right\}$. Thus, $\mathcal{P}=\left(x_{r}, \ldots, x_{1}, x\right)$ contains an oriented cycle $C$. We take a maximal unicycle oriented subgraph $M_{l+1}$ of $K^{l+1}$ with cycle $C, V^{l+1}=C$ and $W_{1}^{l+1}=W_{2}^{l+1}=\emptyset$. Then, $M_{l+1}$ satisfies (a), (b) and (c).

Since $K$ is finite, with this proceeding we obtain $M_{1}, \ldots, M_{t} \subseteq K$ such that $V(K)=$ $\cup_{i=1}^{t} V\left(M_{i}\right), W_{1}^{i} \cap \mathcal{C}=\emptyset$ and $W_{2}^{i} \subseteq\left(\mathcal{C} \backslash L_{1}(\mathcal{C})\right) \cap V^{+}$for $i=1, \ldots t$. We take $H:=\cup_{i=1}^{t} M_{i}$ with $W_{j}=\cup_{i=1}^{t} W_{j}^{i}$ for $j=1,2$. So, $V(H)=V(K)$. Also, $W_{1} \cap \mathcal{C}=\emptyset$, then $W_{1}$ is a stable set, since $\mathcal{C}$ is a vertex cover. Furthermore, $W_{2} \subseteq V^{+}$and $W_{2} \subseteq \mathcal{C} \backslash L_{1}(\mathcal{C})$, then 
$N_{D}^{+}\left(W_{2}\right) \subseteq \mathcal{C}$. Then, $N_{D}^{+}\left(W_{2}\right) \cap W_{1}=\emptyset$, since $\mathcal{C} \cap W_{1}=\emptyset$. If $x \in L_{1}(\mathcal{C}) \cap V(K)$, then there is $1 \leqslant i \leqslant s$ such that $x=v_{i}$ and $M_{i}=\left\{v_{i}\right\}$. Consequently, $\operatorname{deg}_{H}(x)=\operatorname{deg}_{M_{i}}\left(v_{i}\right)=0$. Thus, $\tilde{H} \cap L_{1}(\mathcal{C})=\emptyset$ implying $N_{D}^{+}(\tilde{H}) \subseteq \mathcal{C}$, since $V(H) \subseteq \mathcal{C}$. Hence, $N_{D}^{+}(\tilde{H}) \cap W_{1}=\emptyset$, since $W_{1} \cap \mathcal{C}=\emptyset$. Therefore, $H$ is a generating $\star$-semi-forest of $K$.

Theorem 3.11 Let $D=(G, \mathcal{O}, w)$ be a weighted oriented graph where $G$ is a perfect graph, then $G$ has a $\tau$-reduction $H_{1}, \ldots, H_{s}$ in complete subgraphs. Furthermore, $I(D)$ is unmixed if and only if each $H_{i}$ has no generating $\star$-semi-forests.

Proof. First, we prove $G$ has a $\tau$-reduction in complete graphs. By Theorem $2.34, \bar{G}$ is perfect. Thus, $s:=w(\bar{G})=\chi(\bar{G})$. So, there is a $s$-colouring $c: V(\bar{G}) \rightarrow\{1, \ldots, s\}$. We take $V_{i}:=c^{-1}(i)$ for $i=1, \ldots, s$. Then, $V_{i}$ is a stable set in $\bar{G}$, since $c$ is a $s$ colouring. Hence, by Remark 2.33 $H_{i}:=G\left[V_{i}\right]$ is a complete graph in $G$ and $s=$ $\omega(\bar{G})=\beta(G)$. Furthermore, $V_{1}, \ldots, V_{s}$ is a partition of $V(\bar{G})=V(G)$, since $c$ is a function. Consequently,

$$
\sum_{i=1}^{s} \tau\left(H_{i}\right)=\sum_{i=1}^{s}\left(\left|V_{i}\right|-1\right)=\left(\sum_{i=1}^{s}\left|V_{i}\right|\right)-s=|V(G)|-\beta(G)=\tau(G) .
$$

Finally, by Remark 2.16, $|V(G)|-\beta(G)=\tau(G)$, then, $H_{1}, \ldots, H_{s}$ is a $\tau$-reduction of $G$.

Now, we prove that $I(D)$ is unmixed if and only if each $H_{i}$ has no generating $\star$-semiforests.

$\Rightarrow)$ By contradiction, assume $H_{j}$ has a generating $\star$-semi-forest, then by Theorem 3.10 there is a strong vertex $\mathcal{C}$ such that $V_{j} \subseteq \mathcal{C}$. Furthermore, $\mathcal{C} \cap V_{i}$ is a vertex cover of $H_{i}$, then $\left|\mathcal{C} \cap V_{i}\right| \geqslant \tau\left(H_{i}\right)=\left|V_{i}\right|-1$ for $i \neq j$. Thus, $|\mathcal{C}|=\sum_{i=1}^{s}\left|\mathcal{C} \cap V_{i}\right| \geqslant\left|V_{j}\right|+\sum_{\substack{i=1 \\ i \neq j}}^{s}\left(\left|V_{i}\right|-1\right)$, since $V_{1}, \ldots, V_{s}$ is a partition of $V(G)$. Hence, by Remark 2.16 $|\mathcal{C}|>|V(G)|-s=\tau(G)$, since $s=\beta(G)$. A contradiction, by Remark 2.14, since $I(D)$ is unmixed.

$\Leftarrow)$ Let $\mathcal{C}$ be a strong vertex cover, then $\mathcal{C} \cap V_{i}$ is a vertex cover of $H_{i}$. So, $\left|\mathcal{C} \cap V_{i}\right| \geqslant$ $\tau\left(H_{i}\right)=\left|V_{i}\right|-1$ for $i=1, \ldots, s$. Furthermore, by Theorem 3.10, $V_{i} \nsubseteq \mathcal{C}$. Consequently, $\left|\mathcal{C} \cap V_{i}\right|=\left|V_{i}\right|-1$. Thus, $|\mathcal{C}|=\sum_{i=1}^{s}\left(\left|V_{i}\right|-1\right)$, since $V_{1}, \ldots, V_{s}$ is a partition of $V(G)$. Therefore, by (2) in Theorem 2.12 $I(D)$ is unmixed.

\section{Unmixedness of weighted oriented $S C Q$ graphs}

Let $D=(G, \mathcal{O}, w)$ be a weighted oriented graph. If $P$ is a perfect matching of $G$ with the property $(\mathbf{P})$, then in Proposition 4.1 we characterize when $|\mathcal{C} \cap e|=1$, for each strong vertex cover $\mathcal{C}$ of $D$ and each $e \in P$. Using Proposition 4.1 in Corollary 4.2. we characterize when $I(D)$ is unmixed if $G$ is Köning. In Proposition 4.6, we characterize the basic 5-cycles, $C$ such that $|\mathcal{C} \cap V(C)|=3$ for each strong vertex cover $\mathcal{C}$ of $D$. Furthermore, in Theorem 4.8, we characterize when $I(D)$ is unmixed if $G$ is an $S C Q$ graph (see Definition 2.27). Finally, using this result we characterize the unmixed property of $I(D)$, when $G$ is simplicial or $G$ is chordal (see Corollary 4.9).

Proposition 4.1 Let e be an edge of $G$. Hence, the following conditions are equivalent:

(1) $|\mathcal{C} \cap e|=1$ for each strong vertex cover $\mathcal{C}$ of $D$. 
(2) e has the property $(\mathbf{P})$ and $N_{D}(b) \subseteq N_{D}^{+}(a)$ if $\left(a, b^{\prime}\right) \in E(D)$ with $a \in V^{+}$and $e=\left\{b, b^{\prime}\right\}$.

Proof. (1) $\Rightarrow(2)$ First, we show $e$ has the property (P). By contradiction, suppose there are $\{a, b\},\left\{a^{\prime}, b^{\prime}\right\} \in E(G)$ such that $\left\{a, a^{\prime}\right\} \notin E(G)$. This implies, there is a maximal stable set $S$ such that $\left\{a, a^{\prime}\right\} \subseteq S$. So, $\tilde{\mathcal{C}}=V(G) \backslash S$ is a minimal vertex cover. Consequently, $\tilde{\mathcal{C}}$ is strong. Furthermore, $a, a^{\prime} \notin \tilde{\mathcal{C}}$, then $b, b^{\prime} \in \tilde{\mathcal{C}}$, since $\{a, b\},\left\{a^{\prime}, b^{\prime}\right\} \in$ $E(G)$. A contradiction by (1). Now, assume $\left(a, b^{\prime}\right) \in E(D)$ with $a \in V^{+}$and $e=$ $\left\{b, b^{\prime}\right\}$, then we will prove that $N_{D}(b) \subseteq N_{D}^{+}(a)$. By contradiction, suppose there is $c \in N_{D}(b) \backslash N_{D}^{+}(a)$. We take a maximal stable set $S$ such that $b \in S$. Thus, $\mathcal{C}_{1}=V(G) \backslash S$ is a minimal vertex cover such that $b \notin \mathcal{C}_{1}$. By $\operatorname{Remark} 2.6$, $\mathcal{C}=\left(\mathcal{C}_{1} \backslash\{c\}\right) \cup N_{D}(c) \cup N_{D}^{+}(a)$ is a vertex cover. Furthermore, $c \notin \mathcal{C}$, since $c \notin N_{D}^{+}(a)$. By Proposition 3.1, there is a strong vertex cover $\mathcal{C}^{\prime}$ such that $N_{D}^{+}(a) \subseteq \mathcal{C}^{\prime} \subseteq \mathcal{C}$, since $a \in V^{+}$. Also, $b^{\prime} \in N_{D}^{+}(a) \subseteq \mathcal{C}^{\prime}$ and $c \notin \mathcal{C}^{\prime}$, since $\left(a, b^{\prime}\right) \in E(D)$ and $c \notin \mathcal{C}$. Then, $b \in N_{D}(c) \subseteq \mathcal{C}^{\prime}$. Hence, $\left\{b, b^{\prime}\right\} \subseteq \mathcal{C}^{\prime}$. This is a contradiction, by (1).

$(2) \Rightarrow(1)$ By contradiction, assume there is a strong vertex cover $\mathcal{C}$ of $D$ such that $|\mathcal{C} \cap e| \neq$ 1. So, $|\mathcal{C} \cap e|=2$, since $\mathcal{C}$ is a vertex cover. Hence, by Theorem 3.10, there is a generating $\star$-semi-forest $H$ of $e$. We set $e=\left\{z, z^{\prime}\right\}$. First, assume $H$ is not connected. Then, using the Definition 3.4, we have $H=M_{1} \cup M_{2}$ where $M_{1}=\left\{v_{1}\right\}, M_{2}=\left\{v_{2}\right\}$ and $w_{1}, w_{2} \in W$ such that $w_{i} \in N_{D}\left(v_{i}\right)$ for $i=1,2$. Thus, $\left\{z, z^{\prime}\right\}=\left\{v_{1}, v_{2}\right\}$ and $\left\{w_{1}, w_{2}\right\} \in E(G)$, since $e$ satisfies the property $(\mathbf{P})$. This implies $\left|W_{1} \cap\left\{w_{1}, w_{2}\right\}\right| \leqslant 1$, since $W_{1}$ is a stable set. Hence, we can suppose $w_{2} \in W_{2}$, then $w_{2} \in V^{+}$and $\left(w_{2}, z^{\prime}\right) \in E(D)$. Consequently, by (2), $w_{1} \in N_{D}(z) \subseteq N_{D}^{+}\left(w_{2}\right)$, then $\left(w_{2}, w_{1}\right) \in E(D)$. Furthermore, by (iii) in Definition 3.4 $N_{D}^{+}\left(W_{2}\right) \cap W_{1}=\emptyset$, then $w_{1} \in W_{2}$. So, $w_{1} \in V^{+}$and $\left(w_{1}, z\right) \in E(D)$. By (1) with $a=w_{1}$, we have $\left(w_{1}, w_{2}\right) \in E(D)$. A contradiction, then $H$ is connected. Thus, $H$ is a ROT with $V(H)=\left\{z, z^{\prime}\right\}$. We can suppose $v_{1}=z$ and $W^{H}=\left\{w_{1}\right\}$, then $\left(z, z^{\prime}\right) \in E(D), w_{1} \in N_{D}(z)$ and $z=v_{1} \in \tilde{H}$, since $\operatorname{deg}_{H}\left(v_{1}\right)=1$. If $w_{1} \in N_{D}^{+}(z)$, then $w_{1} \in W_{1}$, since $z=v_{1}$. A contradiction, since $N_{D}^{+}(\tilde{H}) \cap W_{1}=\emptyset$. Then, $w_{1} \notin N_{D}^{+}(z)$. By Remark 3.5. $z=v_{1} \in \tilde{H} \subseteq V^{+}$. Therefore, by (1) (taking $a=b=z$ and $b^{\prime}=z^{\prime}$ ), we have $N_{D}(z) \subseteq N_{D}^{+}(z)$, since $e=\left\{z, z^{\prime}\right\}$ and $z^{\prime} \in N_{D}^{+}(z)$. A contradiction, since $w_{1} \in N_{D}(z) \backslash N_{D}^{+}(z)$.

Corollary 4.2 [11, Theorem 3.4] Let $D=(G, \mathcal{O}, w)$ be a weighted oriented graph, where $G$ is Köning without isolated vertices. Hence, $I(D)$ is unmixed if and only if $D$ satisfies the following two conditions:

(a) $G$ has a perfect matching $P$ with the property $(\mathbf{P})$.

(b) $N_{D}(b) \subseteq N_{D}^{+}(a)$, when $a \in V^{+},\left\{b, b^{\prime}\right\} \in P$ and $b^{\prime} \in N_{D}^{+}(a)$.

Proof. $\Rightarrow$ ) By Theorem 2.12, $I(G)$ is unmixed. Thus, by Remark 2.17 and Theorem 2.20, $G$ has a perfect matching $P$ with the property $(\mathbf{P})$. Consequently, $\nu(G)=|P|$. Also, $\tau(G)=\nu(G)$, since $G$ is Köning. So, $\tau(G)=|P|$. Now, we take a strong vertex cover $\mathcal{C}$ of $D$ and $e \in P$. Then, $|\mathcal{C} \cap e| \geqslant 1$. Furthermore, by Remark 2.14, $|\mathcal{C}|=\tau(G)=|P|$. Hence, $|\mathcal{C} \cap e|=1$, since $\mathcal{C}=\cup_{\tilde{e} \in P} \mathcal{C} \cap \tilde{e}$. Therefore, by Proposition $4.1 D$ satisfies (b).

$\Leftarrow)$ We take a strong vertex cover $\mathcal{C}$ of $D$. By Proposition $4.1|\mathcal{C} \cap e|=1$ for each $e \in P$, since $D$ satisfies (a) and (b). This implies $|\mathcal{C}|=|P|$, since $P$ is a perfect matching. Therefore, by (2) in Theorem 2.12 $I(D)$ is unmixed. 
Lemma 4.3 If there is a basic 5-cycle $C=\left(z_{1}, z_{2}, z_{3}, z_{4}, z_{5}, z_{1}\right)$ with $\left(z_{1}, z_{2}\right),\left(z_{2}, z_{3}\right) \in$ $E(D), z_{2} \in V^{+}$and $C$ satisfies one of the following conditions:

(a) $\left(z_{3}, z_{4}\right) \in E(D)$ with $z_{3} \in V^{+}$.

(b) $\left(z_{1}, z_{5}\right),\left(z_{5}, z_{4}\right) \in E(D)$ with $z_{5} \in V^{+}$.

then there is a strong vertex cover $\tilde{\mathcal{C}}$ such that $|\tilde{\mathcal{C}} \cap V(C)|=4$.

Proof. We take $\mathcal{C}=\left(\mathcal{C}_{0} \backslash V(C)\right) \cup N_{D}\left(z_{1}\right) \cup N_{D}^{+}\left(z_{2}, x\right)$ where $\mathcal{C}_{0}$ is a vertex cover and $x=z_{3}$ if $C$ satisfies (a) or $x=z_{5}$ if $C$ satisfies (b). Thus, $x \in V^{+}$. Furthermore, $z_{2}, z_{3}, z_{5} \in$ $N_{D}\left(z_{1}\right) \cup N_{D}^{+}\left(z_{2}\right)$ and $z_{4} \in N_{D}^{+}\left(z_{3}\right)$ if $C$ satisfies (a) or $z_{4} \in N_{D}^{+}\left(z_{5}\right)$ if $C$ satisfies (b). Hence, $\left\{z_{2}, z_{3}, z_{4}, z_{5}\right\} \subseteq N_{D}\left(z_{1}\right) \cup N_{D}^{+}\left(z_{2}, x\right)$. Consequently, $\left\{z_{2}, z_{3}, z_{4}, z_{5}\right\} \subseteq \mathcal{C}$, implying $\mathcal{C}$ is a vertex cover, since $\mathcal{C}_{0}$ is vertex cover and $N_{D}\left(z_{1}\right) \subseteq \mathcal{C}$. Also, $z_{1} \notin \mathcal{C}$, since $z_{1} \notin N_{D}\left(z_{1}\right) \cup N_{D}^{+}\left(z_{2}, z_{3}\right)$ and $z_{1} \notin N_{D}^{+}\left(z_{5}\right)$ if $C$ satisfies (b). By Proposition 3.1, there is a strong vertex cover $\mathcal{C}^{\prime}$ such that $N_{D}^{+}\left(z_{2}, x\right) \subseteq \mathcal{C}^{\prime} \subseteq \mathcal{C}$, since $\left\{z_{2}, x\right\} \subseteq V^{+}$. So, $z_{1} \notin \mathcal{C}^{\prime}$, since $z_{1} \notin \mathcal{C}$. Then, by Remark [2.8, $N_{D}\left(z_{1}\right) \subseteq \mathcal{C}^{\prime}$. Hence, $\left\{z_{2}, z_{3}, z_{4}, z_{5}\right\} \subseteq$ $N_{D}\left(z_{1}\right) \cup N_{D}^{+}\left(z_{2}, x\right) \subseteq \mathcal{C}^{\prime}$. Therefore, $\left|\mathcal{C}^{\prime} \cap V(C)\right|=4$, since $z_{1} \notin \mathcal{C}^{\prime}$.

Definition 4.4 Let $C$ be an induced 5-cycle, we say that $C$ has the $\star$-property if for each $(a, b) \in E(C)$ where $a \in V^{+}$, then $C=\left(a^{\prime}, a, b, b^{\prime}, c, a^{\prime}\right)$ with the following properties:

$(\star .1)\left(a^{\prime}, a\right) \in E(D)$ and $w\left(a^{\prime}\right)=1$.

$(\star .2) \quad N_{D}^{-}(a) \subseteq N_{D}(c)$ and $N_{D}^{-}(a) \cap V^{+} \subseteq N_{D}^{-}(c)$.

$(\star .3) N_{D}\left(b^{\prime}\right) \subseteq N_{D}\left(a^{\prime}\right) \cup N_{D}^{+}(a)$ and $N_{D}^{-}\left(b^{\prime}\right) \cap V^{+} \subseteq N_{D}^{-}\left(a^{\prime}\right)$.

Lemma 4.5 Let $C=\left(a_{1}^{\prime}, a_{1}, b_{1}, b_{1}^{\prime}, c_{1}, a_{1}^{\prime}\right)$ be a basic 5-cycle of $D$, such that $\left(a_{1}^{\prime}, a_{1}\right) \in$ $E(D), \operatorname{deg}_{D}\left(a_{1}\right) \geqslant 3, \operatorname{deg}_{D}\left(c_{1}\right) \geqslant 3$ and $w\left(b_{1}\right)=1$. If there is a strong vertex cover $\mathcal{C}$ of $D$, such that $V(C) \subseteq \mathcal{C}$, then $C$ has no the $\star$-property.

Proof. By contradiction, suppose $C$ has the $\star$-property and there is a strong vertex cover $\mathcal{C}$, such that $V(C) \subseteq \mathcal{C}$. Then, $\operatorname{deg}_{D}\left(a_{1}^{\prime}\right)=\operatorname{deg}_{D}\left(b_{1}^{\prime}\right)=2$, since $C$ is a basic cycle, $\operatorname{deg}_{D}\left(a_{1}\right) \geqslant 3$ and $\operatorname{deg}_{D}\left(c_{1}\right) \geqslant 3$. Hence, $a_{1}^{\prime}, b_{1}^{\prime} \in L_{3}(\mathcal{C})$, since $V(C) \subseteq \mathcal{C}$. Thus, $\left(c_{1}, a_{1}^{\prime}\right) \in E(D)$ and $w\left(c_{1}\right) \neq 1$, since $a_{1}^{\prime} \in L_{3}(\mathcal{C}), \operatorname{deg}_{D}\left(a_{1}^{\prime}\right)=2,\left(a_{1}^{\prime}, a_{1}\right) \in E(D)$ and $\mathcal{C}$ is strong. By $(\star .1)$ with $(a, b)=\left(c_{1}, a_{1}^{\prime}\right)$, we have that $\left(b_{1}^{\prime}, c_{1}\right) \in E(D)$. Hence, $N_{D}^{-}\left(b_{1}^{\prime}\right) \subseteq\left\{b_{1}\right\}$, since $\operatorname{deg}_{D}\left(b_{1}^{\prime}\right)=2$. This is a contradiction, since $b_{1}^{\prime} \in L_{3}(\mathcal{C})$ and $w\left(b_{1}\right)=1$.

Proposition 4.6 Let $C$ be a basic 5-cycle, then $C$ has the $\star$-property if and only if $|\mathcal{C} \cap V(C)|=3$ for each strong vertex cover $\mathcal{C}$ of $D$.

Proof. $\Rightarrow)$ By contradiction, we suppose there is a strong vertex cover $\mathcal{C}$ such that $|\mathcal{C} \cap V(C)| \geqslant 4$. Thus, there is a path $L=\left(d_{1}, d_{2}, d_{3}, d_{4}\right) \subseteq C$ such that $V(L) \subseteq \mathcal{C}$. Then, $\operatorname{deg}_{D}\left(d_{2}\right)=2$ or $\operatorname{deg}_{D}\left(d_{3}\right)=2$, since $C$ is basic. We can suppose $\operatorname{deg}_{D}\left(d_{2}\right)=2$, then $N_{D}\left(d_{2}\right) \subseteq \mathcal{C}$. This implies $b_{1}:=d_{2} \in L_{3}(\mathcal{C})$. So, there is $\left(a_{1}, b_{1}\right) \in E(D)$ with $a_{1} \in(\mathcal{C} \backslash$ $\left.L_{1}(\mathcal{C})\right) \cap V^{+}$, since $\mathcal{C}$ is strong. Since, $N_{D}\left(b_{1}\right) \subseteq C$, we can set $C=\left(a_{1}^{\prime}, a_{1}, b_{1}, b_{1}^{\prime}, c_{1}, a_{1}^{\prime}\right)$. Consequently, $\left\{a_{1}, b_{1}^{\prime}\right\}=N_{D}\left(b_{1}\right)=N_{D}\left(d_{2}\right)=\left\{d_{1}, d_{3}\right\} \subseteq \mathcal{C}$. By $(\star .1),\left(a_{1}^{\prime}, a_{1}\right) \in E(D)$ and $w\left(a_{1}^{\prime}\right)=1$. If $b_{1} \in V^{+}$, then by Remark [2.3, $b_{1}$ is not a sink. This implies, $\left(b_{1}, b_{1}^{\prime}\right) \in E(D)$. Then, by $(\star .1)$ with $(a, b)=\left(b_{1}, b_{1}^{\prime}\right), w\left(a_{1}\right)=1$. A contradiction, since $a_{1} \in V^{+}$. Hence, $w\left(b_{1}\right)=1$. 
We prove $a_{1}^{\prime} \in \mathcal{C}$. By contradiction assume $a_{1}^{\prime} \notin \mathcal{C}$, then $\left\{b_{1}, a_{1}, c_{1}, b_{1}^{\prime}\right\} \subseteq \mathcal{C}$, since $|\mathcal{C} \cap V(C)| \geqslant 4$. Suppose $b_{1}^{\prime} \in L_{3}(\mathcal{C})$, then there is $y \in\left(N_{D}^{-}\left(b_{1}^{\prime}\right) \cap V^{+}\right) \backslash L_{1}(\mathcal{C})$. Then, by $(\star .3)$ with $(a, b)=\left(a_{1}, b_{1}\right), y \in N_{D}^{-}\left(a_{1}^{\prime}\right)$, i.e. $\left(y, a_{1}^{\prime}\right) \in E(D)$. Consequently, $y \in L_{1}(\mathcal{C})$, since $a_{1}^{\prime} \notin \mathcal{C}$. This is a contradiction. Hence, $b_{1}^{\prime} \notin L_{3}(\mathcal{C})$, i.e. there is $y^{\prime} \in N_{D}\left(b_{1}^{\prime}\right) \backslash \mathcal{C}$, since $b_{1}^{\prime} \in \mathcal{C}$. By $(\star .3), y^{\prime} \in N_{D}\left(a_{1}^{\prime}\right) \cup N_{D}^{+}\left(a_{1}\right)$. Furthermore, $a_{1}^{\prime} \notin \mathcal{C}$, then $N_{D}\left(a_{1}^{\prime}\right) \subseteq \mathcal{C}$ and $y^{\prime} \notin N_{D}\left(a_{1}^{\prime}\right)$, since $\mathcal{C}$ is a vertex cover and $y^{\prime} \notin \mathcal{C}$. This implies $y^{\prime} \in N_{D}^{+}\left(a_{1}\right)$, then $a_{1} \in L_{1}(\mathcal{C})$, since $a_{1} \in \mathcal{C}$ and $y^{\prime} \notin \mathcal{C}$. A contradiction, since $a_{1} \notin L_{1}(\mathcal{C})$. Therefore, $a_{1}^{\prime} \in \mathcal{C}$.

Thus, $\left\{b_{1}, a_{1}, a_{1}^{\prime}, b_{1}^{\prime}\right\} \subseteq \mathcal{C}$. Now, we prove $c_{1} \in \mathcal{C}, \operatorname{deg}_{D}\left(a_{1}\right) \geqslant 3$ and $\operatorname{deg}_{D}\left(c_{1}\right) \geqslant 3$.

Case (1) $a_{1} \in L_{3}(\mathcal{C})$. Consequently, there is $z \in N_{D}^{-}\left(a_{1}\right) \cap V^{+}$such that $z \in \mathcal{C} \backslash L_{1}(\mathcal{C})$. Then, $z \notin V(C)$, since $N_{D}^{-}\left(a_{1}\right) \cap V(C)=\left\{a_{1}^{\prime}\right\}$ and $w\left(a_{1}^{\prime}\right)=1$. By $(\star .2), z \in N_{D}^{-}\left(c_{1}\right)$. Thus, $\left(z, c_{1}\right) \in E(D)$. Consequently, $c_{1} \in \mathcal{C}, \operatorname{deg}_{D}\left(a_{1}\right) \geqslant 3$ and $\operatorname{deg}_{D}\left(c_{1}\right) \geqslant 3$, since $z \in \mathcal{C} \backslash L_{1}(\mathcal{C})$ and $z \in N_{D}\left(a_{1}\right) \cap N_{D}\left(c_{1}\right)$.

Case (2) $a_{1} \notin L_{3}(\mathcal{C})$. This implies, there is $z^{\prime} \in N_{D}\left(a_{1}\right)$ such that $z^{\prime} \notin \mathcal{C}$. Then, $z^{\prime} \notin V(C)$, since $N_{D}\left(a_{1}\right) \cap V(C)=\left\{a_{1}^{\prime}, b_{1}\right\} \subseteq \mathcal{C}$. Consequently, $z^{\prime} \in N_{D}^{-}\left(a_{1}\right)$, since $a_{1} \in \mathcal{C} \backslash L_{1}(\mathcal{C})$. By $(\star .2)$, we have $z^{\prime} \in N_{D}^{-}\left(a_{1}\right) \subseteq N_{D}\left(c_{1}\right)$. Hence, $c_{1} \in \mathcal{C}, \operatorname{deg}_{D}\left(a_{1}\right) \geqslant 3$ and $\operatorname{deg}_{D}\left(c_{1}\right) \geqslant 3$, since $z^{\prime} \notin \mathcal{C}$ and $z^{\prime} \in N_{D}\left(a_{1}\right) \cap N_{D}\left(c_{1}\right)$.

This implies, $V(C) \subseteq \mathcal{C}$. A contradiction, by Lemma 4.5, since $C$ has the $\star$-property.

$\Leftarrow)$ Assume $C=\left(a^{\prime}, a, b, b^{\prime}, c, a^{\prime}\right)$ with $(a, b) \in E(C)$ such that $w(a) \neq 1$. We take a minimal vertex cover $\mathcal{C}$ of $D$. We will prove $(\star .1),(\star .2)$ and $(\star .3)$.

( .1) First we will prove $\left(a^{\prime}, a\right) \in E(D)$. By contradiction, suppose $\left(a, a^{\prime}\right) \in E(D)$. By Remark 2.3, there is $y \in N_{D}^{-}(a)$, since $a \in V^{+}$. Thus, $y \notin V(C)$ and $\operatorname{deg}_{D}(a) \geq 3$. Consequently, $\operatorname{deg}_{D}\left(a^{\prime}\right)=\operatorname{deg}_{D}(b)=2$, since $C$ is basic. Also, $\operatorname{deg}_{D}\left(b^{\prime}\right)=2$ or $\operatorname{deg}_{D}(c)=$ 2 , since $C$ is basic. We can assume $\operatorname{deg}_{D}(c)=2$, then $N_{D}(c)=\left\{a^{\prime}, b^{\prime}\right\}$. So, by Remark 2.6. $\mathcal{C}_{1}=(\mathcal{C} \backslash\{y, c\}) \cup N_{D}(y, b) \cup N_{D}^{+}(a)$ is a vertex cover, since $\mathcal{C}$ is a vertex cover, $\left\{a^{\prime}, b^{\prime}\right\} \subseteq N_{D}(b) \cup N_{D}^{+}(a) \subseteq \mathcal{C}_{1}$. Since $\operatorname{deg}_{D}(c)=2$, we have $c \notin N_{D}(y)$. Furthermore, $c \notin N_{D}(b) \cup N_{D}^{+}(a)$, since $C$ is induced. Then, $c \notin \mathcal{C}_{1}$. Also, $N_{D}(b)=\left\{b^{\prime}, a\right\}$, implies $y \notin \mathcal{C}_{1}$, since $y \notin N_{D}^{+}(a)$. By Proposition 3.1 there is a strong vertex cover $\mathcal{C}_{1}^{\prime}$ such that $N_{D}^{+}(a) \subseteq \mathcal{C}_{1}^{\prime} \subseteq \mathcal{C}_{1}$, since $a \in V^{+}$. Thus, $c, y \notin \mathcal{C}_{1}^{\prime}$, since $c, y \notin \mathcal{C}_{1}$. By Remark 2.8 $a^{\prime}, b^{\prime}, a \in N_{D}(c) \cup N_{D}(y) \subseteq \mathcal{C}_{1}^{\prime}$. Furthermore, $b \in N_{D}^{+}(a) \subseteq \mathcal{C}_{1}^{\prime}$. Hence, $\left|\mathcal{C}_{1}^{\prime} \cap V(C)\right|=4$. A contradiction.

Now, we prove $w\left(a^{\prime}\right)=1$. By contradiction, assume $w\left(a^{\prime}\right) \neq 1$. By the last argument, $\left(c, a^{\prime}\right) \in E(D)$, since $\left(a^{\prime}, a\right) \in E(D)$ and $a \in V^{+}$. A contradiction, by (a) in Lemma 4.3 . ( $\star$.2) We will prove $N_{D}^{-}(a) \subseteq N_{D}(c)$. By contradiction, suppose there is $y \in N_{D}^{-}(a) \backslash$ $N_{D}(c)$. Also, $N_{D}^{-}(a) \cap V(C) \subseteq\left\{a^{\prime}\right\} \subseteq N_{D}(c)$, since $b \in N_{D}^{+}(a)$. Hence, $y \notin V(C)$. By Remark 2.6. $\mathcal{C}_{2}=(\mathcal{C} \backslash\{y, c\}) \cup N_{D}(y, c) \cup N_{D}^{+}(a)$ is a vertex cover. Furthermore, $y, c \notin \mathcal{C}_{2}$, since $y \in N_{D}^{-}(a) \backslash N_{D}(c)$ and $c \notin N_{D}(a, y)$. By Proposition 3.1, there is a strong vertex cover $\mathcal{C}_{2}^{\prime}$ such that $N_{D}^{+}(a) \subseteq \mathcal{C}_{2}^{\prime} \subseteq \mathcal{C}_{2}$, since $a \in V^{+}$. Thus, $y, c \notin \mathcal{C}_{2}^{\prime}$ since $y, c \notin \mathcal{C}_{2}$. By Remark 2.8, $a, a^{\prime}, b^{\prime} \in N_{D}(y, c) \subseteq \mathcal{C}_{2}^{\prime}$. Hence, $\left|\mathcal{C}_{2}^{\prime} \cap V(C)\right|=4$, since $b \in N_{D}^{+}(a) \subseteq \mathcal{C}_{2}^{\prime}$. A contradiction.

Now, we prove $N_{D}^{-}(a) \cap V^{+} \subseteq N_{D}^{-}(c)$. By contradiction, suppose there is $y \in N_{D}^{-}(a) \cap$ $V^{+} \backslash N_{D}^{-}(c)$. By Remark 2.6, $\mathcal{C}_{3}=(\mathcal{C} \backslash\{c\}) \cup N_{D}(c) \cup N_{D}^{+}(a, y)$ is a vertex cover. Furthermore, $c \notin N_{D}^{+}(a, y)$, then $c \notin \mathcal{C}_{3}$. By Proposition 3.1], there is a strong vertex cover $\mathcal{C}_{3}^{\prime}$ such that $N_{D}^{+}(a, y) \subseteq \mathcal{C}_{3}^{\prime} \subseteq \mathcal{C}_{3}$ since $\{a, y\} \subseteq V^{+}$. So, $c \notin \mathcal{C}_{3}^{\prime}$, since $c \notin \mathcal{C}_{3}$. Thus, by Remark $2.8 a^{\prime}, b^{\prime} \in N_{D}(c) \subseteq \mathcal{C}_{3}^{\prime}$. Also, $a, b \in N_{D}^{+}(a, y) \subseteq \mathcal{C}_{3}^{\prime}$. Hence, $\left|\mathcal{C}_{3}^{\prime} \cap V(C)\right|=4$, a contradiction. 
( $\star$.3) We prove $N_{D}\left(b^{\prime}\right) \subseteq N_{D}\left(a^{\prime}\right) \cup N_{D}^{+}(a)$. By contradiction, we suppose there is $y \in$ $N_{D}\left(b^{\prime}\right) \backslash\left(N_{D}\left(a^{\prime}\right) \cup N_{D}^{+}(a)\right)$. Thus, $y \notin C$, since $N_{D}\left(b^{\prime}\right) \cap V(C)=\{c, b\} \subseteq N_{D}\left(a^{\prime}\right) \cup N_{D}^{+}(a)$. By Remark 2.6. $\left.\mathcal{C}_{4}=\left(\mathcal{C} \backslash\left\{y, a^{\prime}\right\}\right)\right) \cup N_{D}\left(y, a^{\prime}\right) \cup N_{D}^{+}(a)$. Furthermore, $y \notin \mathcal{C}_{4}$, since $y \notin N_{D}\left(a^{\prime}\right) \cup N_{D}^{+}(a)$. By $(\star .1),\left(a^{\prime}, a\right) \in E(D)$, then $a^{\prime} \notin \mathcal{C}_{4}$, since $a^{\prime} \notin N_{D}(y) \cup N_{D}^{+}(a)$. By Proposition 3.1, there is a strong vertex cover $\mathcal{C}_{4}^{\prime}$ such that $N_{D}^{+}(a) \subseteq \mathcal{C}_{4}^{\prime} \subseteq \mathcal{C}_{4}$, since $a \in V^{+}$. So, $y, a^{\prime} \notin \mathcal{C}_{4}^{\prime}$, since $y, a^{\prime} \notin \mathcal{C}_{4}$. Thus, by Remark $2.8 b^{\prime}, a, c \in N_{D}(y) \cup N_{D}\left(a^{\prime}\right) \subseteq$ $\mathcal{C}_{4}^{\prime}$. Also, $b \in N_{D}^{+}(a) \subseteq \mathcal{C}_{4}^{\prime}$. Hence, $\left|\mathcal{C}_{4}^{\prime} \cap V(C)\right|=4$, a contradiction.

Finally, we prove $N_{D}^{-}\left(b^{\prime}\right) \cap V^{+} \subseteq N_{D}^{-}\left(a^{\prime}\right)$. By contradiction, we suppose there is $y \in$ $\left(N_{D}^{-}\left(b^{\prime}\right) \cap V^{+}\right) \backslash N_{D}^{-}\left(a^{\prime}\right)$. By $(\star .1), a^{\prime} \in N_{D}^{-}(a)$. Furthermore, by (a) in Lemma 4.3 , $y \neq b$, since $y \in V^{+}$. If $y=c$, then $\left(c, b^{\prime}\right) \in E(D)$. Thus, by $(\star .1)$, with the edge $\left(a^{\prime}, c\right) \in E(D)$. A contradiction by (b) in Lemma 4.3. since $c=y \in V^{+}$. Hence, $y \notin V(C)$. By Remark 2.6. $\mathcal{C}_{5}=\left(\mathcal{C} \backslash\left\{a^{\prime}\right\}\right) \cup N_{D}\left(a^{\prime}\right) \cup N_{D}^{+}(y, a)$ is a vertex cover. By Remark 2.6] $a^{\prime} \notin N_{D}^{+}(y, a)$, since $\left(a^{\prime}, a\right) \in E(D)$ and $y \notin N_{D}^{-}\left(a^{\prime}\right)$. Consequently, $a^{\prime} \notin \mathcal{C}_{5}$. By Proposition 3.1 there is a strong vertex cover $\mathcal{C}_{5}^{\prime}$ such that $N_{D}^{+}(a, y) \subseteq \mathcal{C}_{5}^{\prime} \subseteq \mathcal{C}_{5}$, since $\{a, y\} \subseteq V^{+}$. So, $a^{\prime} \notin \mathcal{C}_{5}^{\prime}$, since $a^{\prime} \notin \mathcal{C}_{5}$. Then, by Remark $2.8 a, c \in N_{D}\left(a^{\prime}\right) \subseteq \mathcal{C}_{5}^{\prime}$. Furthermore, $b, b^{\prime} \in N_{D}^{+}(a, y) \subseteq \mathcal{C}_{5}^{\prime}$. Hence, $\left|\mathcal{C}_{5}^{\prime} \cap V(C)\right|=4$, a contradiction.

Lemma 4.7 Let $\mathcal{C}$ be a vertex cover of $D$ where $G$ is an $S C Q$ graph. Hence, $|\mathcal{C}|=\tau(G)$ if and only if $|\mathcal{C} \cap V(K)|=|V(K)|-1,|\mathcal{C} \cap V(C)|=3$ and $|\mathcal{C} \cap e|=1$ for each $K \in S_{G}$, $C \in C_{G}$ and $e \in Q_{G}$, respectively.

Proof. We set $\mathcal{C}$ a vertex cover of $D, K \in S_{G}, C \in C_{G}$ and $e \in Q_{G}$. Then, there are $y \in V(G)$ and $a, a^{\prime} \in V(C)$ such that $K=G\left[N_{G}[y]\right], \operatorname{deg}_{G}(a)=\operatorname{deg}_{G}\left(a^{\prime}\right)=2$ and $\left\{a, a^{\prime}\right\} \notin E(G)$. We set $A_{K}:=V(K) \backslash\{y\}$ and $B_{C}:=V(C) \backslash\left\{a, a^{\prime}\right\}$. Also, $\mathcal{C} \cap V(K)$ is a vertex cover of $K$, so $|\mathcal{C} \cap V(K)| \geqslant \tau(K)=|V(K)|-1$. Similarly, $|\mathcal{C} \cap V(C)| \geqslant \tau(C)=3$ and $|\mathcal{C} \cap e| \geqslant \tau(e)=1$. Thus,

$|\mathcal{C}|=\sum_{K \in S_{G}}|\mathcal{C} \cap V(K)|+\sum_{C \in C_{G}}|\mathcal{C} \cap V(C)|+\sum_{e \in Q_{G}}|\mathcal{C} \cap e| \geqslant \sum_{K \in S_{G}}(|V(K)|-1)+3\left|C_{G}\right|+\left|Q_{G}\right|$,

since $\mathcal{H}=\left\{V(H) \mid H \in S_{G} \cup C_{G} \cup Q_{G}\right\}$ is a partition of $V(G)$. Now, we take a maximal stable set $S$ contained in $V\left(Q_{G}\right):=\left\{x \in V(G) \mid x \in e\right.$ and $\left.e \in Q_{G}\right\}$. Then, $|S \cap e| \leqslant 1$ for each $e \in Q_{G}$, since $S$ is stable. If $S \cap e=\emptyset$ for some $e=\left\{x_{1}, x_{2}\right\} \in Q_{G}$, then there are $y_{1}, y_{2} \in S$ such that $\left\{x_{1}, y_{1}\right\},\left\{x_{2}, y_{2}\right\} \in E(G)$, since $S$ is maximal. But $Q_{G}$ satisfies the property $(\mathbf{P})$, then $\left\{y_{1}, y_{2}\right\} \in E(G)$. A contradiction, since $S$ is stable. Hence, $|S \cap e|=1$ for each $e \in Q_{G}$. Consequently, $|S|=\left|Q_{G}\right|$ and $\left|S^{\prime}\right|=\left|Q_{G}\right|$, where $S^{\prime}=V\left(Q_{G}\right) \backslash S$. Now, we take

$$
\mathcal{C}\left(S^{\prime}\right)=\left(\bigcup_{K \in S_{G}} A_{K}\right) \cup\left(\bigcup_{C \in C_{G}} B_{C}\right) \cup S^{\prime} .
$$

We prove $\mathcal{C}\left(S^{\prime}\right)$ is a vertex cover of $D$. By contradiction, suppose there is $\hat{e} \in E(G)$ such that $\hat{e} \cap \mathcal{C}\left(S^{\prime}\right)=\emptyset$. We set $z \in \hat{e}$, then $\hat{e}=\left\{z, z^{\prime}\right\}$. If $z \in V(\tilde{K})$ for some $\tilde{K} \in S_{G}$, then $\tilde{K}=G\left[N_{G}[z]\right]$, since $A_{\tilde{K}} \subseteq \mathcal{C}\left(S^{\prime}\right)$ and $z \notin \mathcal{C}\left(S^{\prime}\right)$. So, $z^{\prime} \in N_{G}(z) \subseteq \tilde{K} \backslash\{z\}=$ $A_{\tilde{K}} \subseteq \mathcal{C}\left(S^{\prime}\right)$. A contradiction, since $\hat{e} \cap \mathcal{C}\left(S^{\prime}\right)=\emptyset$. Now, if $z \in V(\tilde{C})$ for some $\tilde{C} \in C_{G}$, then $z \notin B_{\tilde{C}}$. Thus, $\operatorname{deg}_{G}(z)=2$ implying $z^{\prime} \in B_{\tilde{C}} \subseteq \mathcal{C}\left(S^{\prime}\right)$, since $\left\{z, z^{\prime}\right\} \in E(G)$. A contradiction. Then, $\hat{e} \subseteq V\left(Q_{G}\right)$, since $\mathcal{H}$ is a partition of $V(G)$. Also, $\hat{e} \cap S^{\prime}=\emptyset$, this implies $\hat{e} \subseteq V\left(Q_{G}\right) \backslash S^{\prime}=S$. But $S$ is stable. This is a contradiction. Hence, $\mathcal{C}\left(S^{\prime}\right)$ is a vertex cover of $D$. Furthermore, 


$$
\left|\mathcal{C}\left(S^{\prime}\right)\right|=\sum_{K \in S_{G}}\left|A_{K}\right|+\sum_{C \in C_{G}}\left|B_{C}\right|+\left|S^{\prime}\right|=\sum_{K \in S_{G}}(|V(K)|-1)+3\left|C_{G}\right|+\left|Q_{G}\right| .
$$

Thus, $\tau(G)=\sum_{K \in S_{G}}(|V(K)|-1)+3\left|C_{G}\right|+\left|Q_{G}\right|$. Therefore, by (4.1), $|\mathcal{C}|=\tau(G)$ if and only if $|\mathcal{C} \cap V(K)|=|K|-1,|\mathcal{C} \cap V(C)|=3$ and $|\mathcal{C} \cap e|=1$ for each $K \in S_{G}, C \in C_{G}$ and $e \in Q_{G}$, respectively.

Theorem 4.8 Let $D=(G, \mathcal{O}, w)$ be a weighted oriented graph where $G$ is an $S C Q$ graph. Hence, $I(D)$ is unmixed if and only if $D$ satisfies the following conditions:

(a) Each basic 5-cycle of $G$ has the $\star$-property.

(b) Each simplex of $D$ has no generating $\star$-semi-forests.

(c) $N_{D}(b) \subseteq N_{D}^{+}(a)$ when $a \in V^{+},\left\{b, b^{\prime}\right\} \in Q_{G}$ and $b^{\prime} \in N_{D}^{+}(a)$.

Proof. $\Rightarrow)$ We take a strong vertex cover $\mathcal{C}$ of $D$, then by Remark 2.14 $|\mathcal{C}|=\tau(G)$. Consequently, by Lemma 4.7 $|\mathcal{C} \cap V(K)|=|V(K)|-1,|\mathcal{C} \cap V(C)|=3$ and $|\mathcal{C} \cap e|=1$ for each $K \in S_{G}, C \in C_{G}$ and $e \in Q_{G}$. Thus, $V(K) \nsubseteq \mathcal{C}$. Consequently, by Theorem 3.10, $D$ satisfies (b). Furthermore, by Propositions 4.6 and 4.1, $D$ satisfies (a) and (c).

$\Leftarrow)$ Let $\mathcal{C}$ be a strong vertex cover of $D$. By (a) and Proposition 4.6, we have $|\mathcal{C} \cap V(C)|=$ 3 for each $C \in C_{G}$. Furthermore, by (b) and Theorem 3.10, $V(K) \not \subset \mathcal{C}$ for each $K \in S_{G}$. Consequently, $|V(K)|>|\mathcal{C} \cap V(K)| \geqslant \tau(K)=|V(K)|-1$. So, $|\mathcal{C} \cap V(K)|=|V(K)|-1$. Now, if $e \in Q_{G}$, then $e$ has the property $(\mathbf{P})$, since $Q_{G}$ has the property $(\mathbf{P})$. Thus, by (c) and Proposition 4.1, $|\mathcal{C} \cap e|=1$. Hence, by Lemma 4.7 $|\mathcal{C}|=\tau(G)$. Therefore $I(D)$ is unmixed, by (2) in Theorem 2.12 .

Corollary 4.9 Let $D=(G, \mathcal{O}, w)$ be a weighted oriented graph where $G$ is a simplicial or chordal graph. Hence, $I(D)$ is unmixed if and only if $D$ satisfies the following conditions:

(a) Each vertex is in exactly one simplex of $D$.

(b) Each simplex of $D$ has not a generating $\star$-semi-forest.

Proof. $\Rightarrow$ ) By $(3)$ in Theorem 2.12 and Remark 2.17, $G$ is well-covered. Thus, by Theorem 2.26] $G$ satisfies (a). Furthermore, by Remark 2.29, $G$ is an $S C Q$ graph with $C_{G}=Q_{G}=\emptyset$. Hence, by Theorem $4.8 D$ satifies (b).

$\Leftarrow)$ By (a), $\left\{V(H) \mid H \in S_{G}\right\}$ is a partition of $V(G)$. Hence, $G$ is an $S C Q$ graph with $C_{G}=\emptyset$ and $Q_{G}=\emptyset$. Therefore, by (b) and Theorem 4.8 $I(D)$ is unmixed.

\section{Unmixedness of weighted oriented graphs without some small cycles}

Let $D=(G, \mathcal{O}, w)$ be a weighted oriented graph. In this Section, we study and characterize the unmixed property of $I(D)$ when $G$ has no 3 - or 5- cycles (Theorem 5.4), or $G$ is a graph without 4 - or 5 -cycles (Theorem 5.10), or $\operatorname{girth}(G) \geqslant 5$ (Theorem 5.13). In other words, in this Section, we characterize the unmixed property of $I(D)$ when $G$ has at most one of the following types of cycles: 3-cycles, 4-cycles and 5-cycles.

Proposition 5.1 If for each $(y, x) \in E(D)$ with $y \in V^{+}$, we have that $N_{D}\left(y^{\prime}\right) \subseteq N_{D}^{+}(y)$ for some $y^{\prime} \in N_{D}(x) \backslash y$, then $L_{3}(\mathcal{C})=\emptyset$ for each strong vertex cover $\mathcal{C}$ of $D$. 
Proof. By contradiction, suppose there is a strong vertex cover $\mathcal{C}$ of $D$ and $x \in L_{3}(\mathcal{C})$. Hence, there is $y \in\left(\mathcal{C} \backslash L_{1}(\mathcal{C})\right) \cap V^{+}$with $(y, x) \in E(D)$. Then, $N_{D}(x) \subseteq \mathcal{C}$ and $N_{D}^{+}(y) \subseteq \mathcal{C}$, since $x \in L_{3}(\mathcal{C})$ and $y \in \mathcal{C} \backslash L_{1}(\mathcal{C})$. By hypothesis, there is a vertex $y^{\prime} \in N_{D}(x) \backslash y \subseteq \mathcal{C}$ such that $N_{D}\left(y^{\prime}\right) \subseteq N_{D}^{+}(y) \subseteq \mathcal{C}$. Thus, $y^{\prime} \in L_{3}(\mathcal{C})$. Since $\mathcal{C}$ is strong, there is $\left(y_{1}, y^{\prime}\right) \in E(D)$ with $y_{1} \in V^{+}$. So, $y_{1} \in N_{D}\left(y^{\prime}\right) \subseteq N_{D}^{+}(y)$. On the other hand, $\left(y_{1}, x_{1}\right) \in E(D)$ where $x_{1}:=y^{\prime}$ and $y_{1} \in V^{+}$, then by hypothesis, there is $y_{1}^{\prime} \in N_{D}\left(x_{1}\right) \backslash y_{1}$ such that $N_{D}\left(y_{1}^{\prime}\right) \subseteq N_{D}^{+}\left(y_{1}\right)$. Hence, $y_{1}^{\prime} \in N_{D}\left(x_{1}\right)=N_{D}\left(y^{\prime}\right) \subseteq N_{D}^{+}(y)$. Consequently, $y \in N_{D}\left(y_{1}^{\prime}\right) \subseteq N_{D}^{+}\left(y_{1}\right)$. A contradiction, since $y_{1} \in N_{D}^{+}(y)$.

Corollary 5.2 If $G$ is well-covered and $V^{+}$is a subset of sinks, then $I(D)$ is unmixed.

Proof. If $y \in V^{+}$, then $y$ is a sink. Thus, $(y, x) \notin E(D)$ for each $x \in V(D)$. Hence, by Proposition 5.1 $L_{3}(\mathcal{C})=\emptyset$, for each strong vertex cover $\mathcal{C}$ of $D$. Furthermore, by Remark 2.17 $I(G)$ is unmixed. Therefore $I(D)$ is unmixed, by (3) in Theorem 2.12.

Lemma 5.3 Let $(z, y),(y, x)$ be edges of $D$ with $y \in V^{+}$and $N_{D}(x)=\left\{y, x_{1}, \ldots, x_{s}\right\}$. If there are $z_{i} \in N_{D}\left(x_{i}\right) \backslash N_{D}^{+}(y)$ such that $\left\{z, x, z_{1}, \ldots, z_{s}\right\}$ is a stable set, then $I(D)$ is mixed.

Proof. We take $A:=\left\{z, z_{1}, \ldots, z_{s}\right\}$, then $A \cup\{x\}$ is a stable set. We can take a maximal stable set $S$ of $V(G)$, such that $A \cup\{x\} \subseteq S$. So, $\tilde{\mathcal{C}}=V(G) \backslash S$ is a minimal vertex cover of $D$. Hence, $\mathcal{C}=\tilde{\mathcal{C}} \cup N_{D}^{+}(y)$ is a vertex cover of $D$. Also $A \cap \mathcal{C}=\emptyset$, since $A \subseteq S$, $z \in N_{D}^{-}(y)$ and $z_{i} \notin N_{D}^{+}(y)$. By Proposition 3.1. there is a strong vertex cover $\mathcal{C}^{\prime}$ of $D$ such that $N_{D}^{+}(y) \subseteq \mathcal{C}^{\prime} \subseteq \mathcal{C}$, since $y \in V^{+}$. Thus, $A \cap \mathcal{C}^{\prime}=\emptyset$, since $A \cap \mathcal{C}=\emptyset$. Then, by Remark 2.8, $N_{D}(A) \subseteq \mathcal{C}^{\prime}$. Furthermore $N_{D}(x)=\left\{y, x_{1}, \ldots, x_{s}\right\} \subseteq N_{D}(A)$. Consequently, $N_{D}(x) \subseteq \mathcal{C}^{\prime}$. Hence, $x \in L_{3}\left(\mathcal{C}^{\prime}\right)$, since $x \in N_{D}^{+}(y) \subseteq \mathcal{C}^{\prime}$. Therefore, by (3) in Theorem 2.12, $I(D)$ is mixed.

Theorem 5.4 Let $D=(G, \mathcal{O}, w)$ be a weighted oriented graph such that $G$ has no 3-or 5 -cycles. Hence, $I(D)$ is unmixed if and only if $D$ satisfies the following conditions:

(a) $G$ is well-covered.

(b) If $(y, x) \in E(D)$ with $y \in V^{+}$, then $N_{D}\left(y^{\prime}\right) \subseteq N_{D}^{+}(y)$ for some $y^{\prime} \in N_{D}(x) \backslash y$.

Proof. $\Leftarrow)$ By Proposition 5.1 and $(\mathrm{b})$, we have that $L_{3}(\mathcal{C})=\emptyset$ for each strong vertex cover $\mathcal{C}$ of $D$. Furthermore, by (a) and Remark 2.17, $I(G)$ is unmixed. Therefore, by (3) in Theorem 2.12 $I(D)$ is unmixed.

$\Rightarrow)$ By (3) in Theorem 2.12 and Remark 2.17, $D$ satisfies (a). Now, we take $(y, x) \in E(D)$ with $y \in V^{+}$. Then, by Remark 2.3 , there is $z \in N_{D}^{-}(y)$. Furthermore $z \notin N_{D}(x)$, since $G$ has no 3 -cycles. We set $N_{D}(x) \backslash y=\left\{x_{1}, \ldots, x_{s}\right\}$. We will prove (b). By contradiction, suppose there is $z_{i} \in N_{D}\left(x_{i}\right) \backslash N_{D}^{+}(y)$ for each $i=1, \ldots, s$. If $\left\{z_{i}, z_{j}\right\} \in E(G)$ for some $1 \leqslant i<j \leqslant s$, then $\left(x, x_{i}, z_{i}, z_{j}, x_{j}, x\right)$ is a 5 -cycle. But $G$ has no 5 -cycles, then $\left\{z_{1}, \ldots, z_{s}\right\}$ is a stable set. Now, if $\left\{x, z_{k}\right\} \in E(G)$ or $\left\{z, z_{k}\right\} \in E(G)$ for some $k \in\{1, \ldots, s\}$, then $\left(x, x_{k}, z_{k}, x\right)$ is a 3 -cycle or $\left(z, y, x, x_{k}, z_{k}, z\right)$ is a 5 -cycle. Hence, $\left\{x, z, z_{1}, \ldots, z_{s}\right\}$ is a stable set. A contradiction, by Lemma 5.3 since $I(D)$ is unmixed.

In the following results, we use the notation of Figure 1

Remark 5.5 Let $G$ be a graph in $\left\{C_{7}, T_{10}, P_{10}, P_{13}, P_{14}, Q_{13}\right\}$. Hence, 
(a) $G$ does not contain 4-cycles. Furthermore, if $G$ has a 3-cycle, then $G=T_{10}$.

(b) If $\operatorname{deg}_{G}(x)=2$, then $x$ is not in a 3-cycle of $G$.

(c) If $G \neq C_{7}$ and $\tilde{e}=\{v, u\} \in E(G)$ with $\operatorname{deg}_{D}(v)=\operatorname{deg}_{D}(u)=2$, then $\tilde{e} \in$ $\left\{\tilde{e}_{1}, \tilde{e}_{2}, \tilde{e}_{3}\right\}$. Also, if $\tilde{e}$ is in a 5-cycle $C$, then $G \in\left\{P_{10}, P_{13}\right\}, \tilde{e} \in\left\{\tilde{e}_{1}, \tilde{e}_{2}\right\}$ and $C \in\left\{C_{1}, C_{2}\right\}$ or $G=Q_{13}, \tilde{e}=\tilde{e}_{1}$ and $C=C_{1}$.

(d) If $P=\left(y_{1}, y_{2}, y_{3}\right)$ is a path in $G$ with $\operatorname{deg}_{G}\left(y_{i}\right)=2$ for $i=1,2,3$, then $G=C_{7}$.

Proof. (a) By Theorems 2.28 and 2.30, $G$ has no 4-cycles. Now, if $G$ has a 3-cycle then, by Theorem 2.30, $G=T_{10}$.

(b) By (a), the unique 3-cycle is $\left(c_{1}, c_{2}, c_{3}, c_{1}\right)$ in $T_{10}$ and $\operatorname{deg}_{T_{10}}\left(c_{i}\right)=3$ for $i=1,2,3$.

(c) By Figure 1. $\tilde{e} \in\left\{\tilde{e}_{1}, \tilde{e}_{2}, \tilde{e}_{3}\right\}$ and $G \in\left\{T_{10}, P_{10}, P_{13}, Q_{13}\right\}$, since $G \neq C_{7}$. Now, assume $\tilde{e}$ is in a 5-cycle $C$. By Theorem 2.28, $G \neq T_{10}$. If $G=P_{10}$, then $\tilde{e}_{3}$ is not in a 5-cycle. Thus, $\tilde{e} \in\left\{\tilde{e}_{1}, \tilde{e}_{2}\right\}$ and $C \in\left\{C_{1}, C_{2}\right\}$. Now, if $G=P_{13}$, then $\tilde{e} \in\left\{\tilde{e}_{1}, \tilde{e}_{2}\right\}$ and $C \in\left\{C_{1}, C_{2}\right\}$. Finally, if $G=Q_{13}$, then $\tilde{e}_{2}, \tilde{e}_{3}$ are not in a 5-cycle. Hence, $\tilde{e}=\tilde{e}_{1}$ and $C=C_{1}$.

(d) By contradiction, suppose $G \neq C_{7}$. If $e \in E(P)$, then by (c), $e \in\left\{e_{1}, e_{2}, e_{3}\right\}$. But, $e_{i} \cap e_{j}=\emptyset$ for $i \neq j$. A contradiction, since $P$ is a path.

Lemma 5.6 Let $G$ be a graph in $\left\{C_{7}, T_{10}, P_{10}, P_{13}, P_{14}, Q_{13}\right\}$ with $I(D)$ unmixed. If $(z, y),(y, x) \in E(D)$ with $y \in V^{+}$and $N_{D}(x) \backslash\{y\}=\left\{x_{1}\right\}$, then $\operatorname{deg}_{D}\left(x_{1}\right)=2$.

Proof. By contradiction, suppose $\operatorname{deg}_{D}\left(x_{1}\right) \geqslant 3$. Hence, there are $z_{1}, z_{1}^{\prime} \in N_{D}\left(x_{1}\right) \backslash\{x\}$. By hypothesis, $\operatorname{deg}_{D}(x)=2$. Then, by (b) in Remark [5.5] $x$ is not in a 3 -cycle. So, $x_{1} \neq z$. Furthermore, by (a) in Remark [5.5, $G$ has no 4-cycles. Thus, $z \notin\left\{z_{1}^{\prime}, z_{1}\right\}$ and $z_{1}, z_{1}^{\prime} \notin N_{D}(y)$. If $z_{1}^{\prime}, z_{1} \in N_{D}(z)$, then $\left(x_{1}, z_{1}^{\prime}, z, z_{1}, x_{1}\right)$ is a 4-cycle. A contradiction, then we can assume $z_{1} \notin N_{D}(z)$. Consequently, $\left\{x, z, z_{1}\right\}$ is a stable set, since $x$ is not in a 3-cycle. A contradiction, by Lemma [5.3, since $I(D)$ is unmixed.

Lemma 5.7 If $I(D)$ is unmixed, $G \in\left\{C_{7}, T_{10}, P_{10}, P_{13}, P_{14}, Q_{13}\right\}$ and $\tilde{e}=(y, x) \in E(D)$ with $\operatorname{deg}_{D}(x)=2$ and $y \in V^{+}$, then $G=P_{10}$ and $\tilde{e}=\left(d_{i}, b_{j}\right)$ with $\{i, j\}=\{1,2\}$.

Proof. By Remark 2.3. there is $z \in N_{D}^{-}(y)$. We set $N_{D}(x)=\left\{y, x_{1}\right\}$, then by (b) in Remark [5.5, $z \neq x_{1}$. Thus, by Lemma [5.6] $\operatorname{deg}_{D}\left(x_{1}\right)=2$. Now, we set $N_{D}\left(x_{1}\right)=\left\{x, z_{1}\right\}$. By (b) in Remark [5.5, $x$ is not in a 3 -cycle. So, $z, z_{1} \notin N_{D}(x)$ and $z_{1} \neq y$. Also, by (a) in Remark 5.5. $G$ has no 4-cycles. Then, $z_{1} \neq z$ and $z_{1} \notin N_{D}(y)$. If $z \notin N_{D}\left(z_{1}\right)$, then $\left\{x, z, z_{1}\right\}$ is a stable set. A contradiction, by Lemma 5.3. since $I(D)$ is unmixed. Hence, $\left\{z_{1}, z\right\} \in E(G)$ and $C:=\left(z, y, x, x_{1}, z_{1}, z\right)$ is a 5-cycle. Suppose $y^{\prime} \in N_{D}^{-}(y) \backslash\{z\}$, then $y^{\prime} \notin N_{D}\left(z_{1}\right)$, since $\left(z_{1}, z, y, y^{\prime}, z_{1}\right)$ is not in a 4-cycle in $G$. Consequently, $\left\{y^{\prime}, x, z_{1}\right\}$ is a stable set, since $\operatorname{deg}_{D}(x)=2$. A contradiction, by Lemma 5.3 since $\left(y^{\prime}, y\right),(y, x) \in E(D)$, $y \in V^{+}, N_{D}(x)=\left\{y, x_{1}\right\}$ and $z_{1} \in N_{D}\left(x_{1}\right) \backslash N_{D}^{+}(y)$. Hence, $N_{D}^{-}(y)=\{z\}$. Now, by (c) in Remark 5.5 and by symmetry of $P_{10}$ and $P_{13}$, we can assume $\left\{x, x_{1}\right\}=\tilde{e}_{1}, C=C_{1}$ and $G \in\left\{P_{10}, P_{13}, Q_{13}\right\}$.

First, assume $G=P_{13}$. By symmetry and notation of Figure 1, we can suppose $x_{1}=a_{1}$ and $x=a_{2}$, since $\tilde{e}_{1}=\left\{x, x_{1}\right\}$. Then, $y=b_{2}, z=c_{1}$ and $z_{1}=b_{1}$. Thus, $\left(b_{2}, d_{2}\right) \in$ $E(D)$, since $y=b_{2}$ and $N_{D}^{-}(y)=\{z\}=\left\{c_{1}\right\}$. By Figure 1 $\left(c_{1}, b_{2}\right)=(z, y) \in E(D)$, $\left(b_{2}, d_{2}\right) \in E(D), b_{2}=y \in V^{+}$and $N_{D}\left(d_{2}\right)=\left\{b_{2}, b_{4}, v\right\}$. Also, $a_{4} \in N_{D}\left(b_{4}\right), d_{1} \in N_{D}(v) \backslash$ $N_{D}\left(b_{2}\right)$ and $\left\{c_{1}, d_{2}, a_{4}, d_{1}\right\}$ is a stable set. A contradiction, by Lemma 5.3. since $I(D)$ is unmixed. 
Now, suppose $G=Q_{13}$. By the symmetry of we can suppose $x=a_{2}$ and $x_{1}=a_{1}$, then $d_{2}=y \in V^{+}, z=h$ and $\left(h, d_{2}\right)=(z, y) \in E(D)$. So, $\left(d_{2}, c_{2}\right) \in E(D)$, since $N_{D}^{-}\left(d_{2}\right)=$ $N_{D}^{-}(y)=\{z\}=\{h\}$. A contradiction, by Lemma 5.3, since $\left(h, d_{2}\right),\left(d_{2}, c_{2}\right) \in E(D)$, $d_{2} \in V^{+}, N_{D}\left(c_{2}\right)=\left\{d_{2}, b_{1}\right\}, g_{1} \in N_{D}\left(b_{1}\right) \backslash N_{D}^{+}\left(d_{2}\right)$ and $\left\{h, c_{2}, g_{1}\right\}$ is a stable set.

Hence, $G=P_{10}$ and $\left\{x, x_{1}\right\}=\tilde{e}_{1}=\left\{a_{1}, b_{1}\right\}$. If $x=a_{1}$ and $x_{1}=b_{1}$, then $g_{1}=y \in V^{+}$, $\left(d_{1}, g_{1}\right)=(z, y) \in E(D)$, since $C=C_{1}$. Furthermore, $\left(g_{1}, c_{1}\right) \in E(D)$, since $N_{D}^{-}\left(g_{1}\right)=$ $N_{D}^{-}(y)=\{z\}=\left\{d_{1}\right\}$. A contradiction by Lemma 5.3, since $\left(d_{1}, g_{1}\right),\left(g_{1}, c_{1}\right) \in E(D)$, $g_{1} \in V^{+}, N_{D}\left(c_{1}\right)=\left\{g_{1}, c_{2}\right\}, g_{2} \in N_{D}\left(c_{2}\right)$ and $\left\{d_{1}, c_{1}, g_{2}\right\}$ is stable. Therefore, $x=b_{1}$ and $x_{1}=a_{1}$, implying $y=d_{2}$ and $(y, x)=\left(d_{2}, b_{1}\right)$, since $C=C_{1}$.

Remark 5.8 Assume $I(D)$ is unmixed, $G \in\left\{C_{7}, T_{10}, Q_{13}, P_{13}, P_{14}\right\}, \mathcal{C}$ is a strong vertex cover of $D$ and $y \in \mathcal{C} \cap V^{+}$such that $N_{G}(y) \backslash \mathcal{C} \subseteq V_{2}:=\left\{a \in V(G) \mid \operatorname{deg}_{G}(a)=2\right\}$. We take $b \in N_{G}(y) \backslash \mathcal{C}$. If $(y, b) \in E(D)$, then by Lemma 5.7, $G=P_{10}$. A contradiction, then $(b, y) \in E(D)$. Consequently, $N_{D}(y) \backslash \mathcal{C} \subseteq N_{D}^{-}(y)$, i.e. $N_{D}^{+}(y) \subseteq \mathcal{C}$. Hence, $y \in \mathcal{C} \backslash L_{1}(\mathcal{C})$.

Proposition 5.9 If $I(D)$ is unmixed, with $G \in\left\{C_{7}, T_{10}, Q_{13}, P_{13}, P_{14}\right\}$, then the vertices of $V^{+}$are sinks.

Proof. By contradiction, suppose there is $(y, x) \in E(D)$ with $y \in V^{+}$. Then, by Lemma 5.7. $\operatorname{deg}_{D}(x) \geqslant 3$. Thus, $G \neq C_{7}$. By Remark 2.3, $y$ is not a source. So, there is $(z, y) \in E(D)$. We set $V_{2}:=\left\{a \in V(G) \mid \operatorname{deg}_{G}(a)=2\right\}$. By Theorem 2.12, $L_{3}(\tilde{\mathcal{C}})=\emptyset$ for each strong vertex cover $\tilde{\mathcal{C}}$ of $D$, since $I(D)$ is unmixed. Hence, to obtain a contradiction, we will give a vertex cover $\mathcal{C}$ of $D$ such that $L_{3}(\mathcal{C})=\{x\}$ and $y \in \mathcal{C} \backslash L_{1}(\mathcal{C})$, since with these conditions $\mathcal{C}$ is strong. We will use the notation of Figure 1.

Case (1) If $D=T_{10}$, then $x \in\left\{c_{1}, c_{2}, c_{3}, v\right\}$, since $\operatorname{deg}_{G}(x) \geqslant 3$.

Case (1.a) $x \in\left\{c_{1}, c_{2}, c_{3}\right\}$. By symmetry of $P_{10}$, we can assume $x=c_{1}$ and $y \in\left\{b_{1}, c_{2}\right\}$. Thus, $\mathcal{C}_{1}=\left\{v, a_{2}, a_{3}, b_{1}, c_{1}, c_{2}, c_{3}\right\}$ is a vertex cover with $L_{3}\left(\mathcal{C}_{1}\right)=\{x\}$. If $y=b_{1}$, then $y \in C_{1}$ and $z=a_{1}$, since $N_{D}\left(b_{1}\right)=\left\{a_{1}, c_{1}\right\}$. So, $N_{D}^{+}(y)=\left\{c_{1}\right\} \subseteq \mathcal{C}_{1}$. Now, if $y=c_{2}$, then $y \in \mathcal{C}_{1}$. Furthermore, by Lemma 5.7. $\left(b_{2}, c_{2}\right) \in E(D)$, since $c_{2}=y \in V^{+}, b_{2} \in V_{2}$ and $I(D)$ is unmixed. Consequently, $N_{D}^{+}(y) \subseteq\left\{c_{1}, c_{3}\right\} \subseteq \mathcal{C}_{1}$. Hence, $y \in \mathcal{C}_{1} \backslash L_{1}\left(\mathcal{C}_{1}\right)$ and we take $\mathcal{C}=\mathcal{C}_{1}$.

Case (1.b) $x=v$. Then, $\mathcal{C}_{2}=\left\{v, a_{1}, a_{2}, a_{3}, c_{1}, c_{2}, c_{3}\right\}$ is a vertex cover with $L_{3}\left(\mathcal{C}_{2}\right)=\{x\}$. By symmetry of $P_{10}$, we can suppose $y=a_{1}$. Consequently, $z=b_{1}$ and $N_{D}^{+}(y)=\{v\} \subseteq$ $\mathcal{C}_{2}$, since $a_{1} \in V_{2}$. Hence, $y \in \mathcal{C}_{2} \backslash L_{1}\left(\mathcal{C}_{2}\right)$ and we take $\mathcal{C}=\mathcal{C}_{2}$.

Case (2) If $D=P_{14}$, then, by symmetry, we can assume $y=a_{1}$ and $x \in\left\{a_{2}, b_{1}\right\}$.

Case (2.a) $x=a_{2}$. Thus, $z \in\left\{a_{7}, b_{1}\right\}$. We take $\mathcal{C}_{3}=\left\{a_{1}, a_{2}, a_{3}, a_{4}, a_{6}, b_{1}, b_{2}, b_{5}, b_{6}, b_{7}\right\}$ if $z=a_{7}$ or $\mathcal{C}_{3}=\left\{a_{1}, a_{2}, a_{3}, a_{5}, a_{7}, b_{2}, b_{3}, b_{4}, b_{5}, b_{6}\right\}$ if $z=b_{1}$. So, $\mathcal{C}_{3}$ is a vertex cover of $D, y \in \mathcal{C}_{3}$ and $L_{3}\left(\mathcal{C}_{3}\right)=\{x\}$. Furthermore, $N_{D}(y) \backslash \mathcal{C}_{3}=\{z\}$ and $z \in N_{D}^{-}(y)$, then $y \in \mathcal{C}_{3} \backslash L_{1}\left(\mathcal{C}_{3}\right)$ and we take $\mathcal{C}=\mathcal{C}_{3}$.

Case (2.b) $x=b_{1}$. By symmetry of $P_{14}$, we can suppose $z=a_{2}$. Then, $\overline{\mathcal{C}_{4}}=\left\{a_{1}, a_{3}, a_{4}, a_{5}, a_{7}, b_{1}, b_{2}, b_{3}, b_{6}, b_{7}\right\}$ is a vertex cover of $D$ with $L_{3}\left(\mathcal{C}_{4}\right)=\{x\}$. Also, $N_{D}(y)=\backslash \mathcal{C}_{4}=\left\{a_{2}\right\}$ and $a_{2}=z \in N_{D}^{-}(y)$. Hence, $y \in \mathcal{C}_{4} \backslash L_{1}\left(\mathcal{C}_{4}\right)$ and we take $\mathcal{C}=\mathcal{C}_{4}$.

Case (3) If $D=P_{13}$, then we can assume $x \in\left\{b_{1}, c_{2}, d_{1}\right\}$, since $\operatorname{deg}_{G}(x) \geqslant 3$.

Case (3.a) $x=c_{2}$. Then, $y \in N_{D}\left(c_{2}\right)=\left\{b_{3}, b_{4}, c_{1}\right\}$. Without loss of generality, we can suppose $y \in\left\{b_{3}, c_{1}\right\}$. If $y=c_{1}$, then $z \in\left\{b_{1}, b_{2}\right\}$. By symmetry, we can assume $z=b_{1}$ so $N_{D}^{+}(y)=N_{D}^{+}\left(c_{1}\right) \subseteq\left\{b_{2}, c_{2}\right\}$. We take $\mathcal{C}_{5}=\left\{a_{1}, a_{4}, b_{2}, b_{3}, b_{4}, c_{1}, c_{2}, d_{1}, v\right\}$. Thus, $\mathcal{C}_{5}$ 
is a vertex cover of $D$ with $L_{3}\left(\mathcal{C}_{5}\right)=\{x\}, y \in \mathcal{C}_{5}$ and $N_{D}^{+}(y) \subseteq\left\{b_{2}, c_{2}\right\} \subseteq \mathcal{C}_{5}$. Now, if $y=b_{3}$, then $b_{3} \in V^{+} \cap \mathcal{C}_{5}$. Hence, by Lemma 5.7 $\left(a_{3}, b_{3}\right) \in E(D)$, since $a_{3} \in V_{2}$ and $I(D)$ is unmixed. Consequently, $N_{D}^{+}(y) \subseteq\left\{c_{2}, d_{1}\right\} \subset \mathcal{C}_{5}$. Therefore, $y \in \mathcal{C}_{5} \backslash L_{1}\left(\mathcal{C}_{5}\right)$ and we take $\mathcal{C}=\mathcal{C}_{5}$.

Case (3.b) $x=b_{1}$. Hence, $\mathcal{C}_{6}=\left\{a_{1}, a_{3}, b_{1}, b_{2}, b_{3}, b_{4}, c_{1}, d_{1}, d_{2}\right\}$ is a vertex cover of $D$ with $L_{3}\left(\mathcal{C}_{6}\right)=\{x\}$ and $y \in N_{D}\left(b_{1}\right)=\left\{a_{1}, c_{1}, d_{1}\right\}$. Also, $y \in \mathcal{C}_{6}$. If $y \in\left\{a_{1}, d_{1}\right\}$, then $N_{D}(y) \backslash \mathcal{C}_{6} \subseteq\left\{a_{2}, v\right\} \subseteq V_{2}$. Consequently, by Lemma 5.7. $(b, y) \in E(D)$ for each $b \in N_{D}(y) \backslash \mathcal{C}_{6}$, since $y \in V^{+}$. So, $N_{D}^{+} \subseteq \mathcal{C}_{6}$ implying $y \in \mathcal{C}_{6} \backslash L_{1}\left(\mathcal{C}_{6}\right)$. Now, if $y=c_{1}$. We can assume $\left(c_{2}, c_{1}\right) \in E(D)$, since in another case we have the case (3.a) with $x=c_{2}$ and $y=c_{1}$. Thus, $y=c_{1} \in \mathcal{C}_{6} \backslash L_{1}\left(\mathcal{C}_{6}\right)$, since $N_{D}^{+}\left(c_{1}\right) \subseteq\left\{b_{1}, b_{2}\right\} \subset \mathcal{C}_{6}$. Therefore, we take $\mathcal{C}=\mathcal{C}_{6}$.

Case (3.c) $x=d_{1}$. Then, $y \in N_{G}\left(d_{1}\right)=\left\{b_{1}, b_{3}, v\right\}$. By symmetry, we can assume

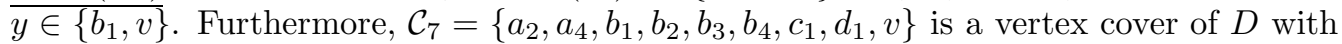
$L_{3}\left(\mathcal{C}_{7}\right)=\{x\}$ and $y \in \mathcal{C}_{7}$. If $y=b_{1}$, then $N_{D}(y) \backslash \mathcal{C}_{7} \subseteq\left\{a_{1}\right\}$. Also, by Lemma 5.7 $N_{D}(y) \backslash \mathcal{C}_{7} \subseteq N_{D}^{-}(y)$, since $a_{1} \in V_{2}$ and $y \in V^{+}$. Thus, $y \in \mathcal{C}_{7} \backslash L_{1}\left(\mathcal{C}_{7}\right)$. Now, if $y=v$, then $z=d_{2}$, since $N_{D}(v)=\left\{d_{1}, d_{2}\right\}$. This implies $N_{D}^{+}(v)=\left\{d_{1}\right\} \subseteq \mathcal{C}_{7}$. So, $y \in \mathcal{C}_{7} \backslash L_{1}\left(\mathcal{C}_{7}\right)$ and we take $\mathcal{C}=\mathcal{C}_{7}$.

Case (4) $D=Q_{13}$. Hence, $x \in\left\{d_{1}, d_{2}, g_{1}, g_{2}, h, h^{\prime}\right\}$, since $\operatorname{deg}_{D}(x) \geqslant 3$. By symmetry, we can suppose $x \in\left\{d_{2}, g_{2}, h, h^{\prime}\right\}$.

Case (4.a) $x \in\left\{d_{2}, g_{2}\right\}$. We take $\mathcal{C}_{8}=\left\{a_{2}, c_{1}, c_{2}, d_{1}, d_{2}, g_{1}, g_{2}, h, h^{\prime}\right\}$ if $x=d_{2}$ or $\mathcal{C}_{8}=$ $\left.\overline{\left\{a_{1}, b_{2}, c_{2},\right.} d_{1}, d_{2}, g_{1}, g_{2}, h, h^{\prime}\right\}$ if $x=g_{2}$. Thus, $\mathcal{C}_{8}$ is a vertex cover of $D$ with $L_{3}\left(\mathcal{C}_{8}\right)=\{x\}$ and $V(G) \backslash \mathcal{C}_{8}=\left\{a_{1}, b_{1}, b_{2}, v\right\} \cup\left\{a_{2}, b_{1}, c_{1}, v\right\} \subseteq V_{2}$. Consequently, by Lemma 5.7 $N_{D}(y) \backslash \mathcal{C}_{8} \subseteq N_{D}^{-}(y)$, since $y \in V^{+}$. This implies $N_{D}^{+}(y) \subseteq \mathcal{C}_{8}$. Therefore $y \in \mathcal{C}_{8} \backslash L_{1}\left(\mathcal{C}_{8}\right)$ and we take $\mathcal{C}=\mathcal{C}_{8}$.

Case (4.b) $x \in\left\{h, h^{\prime}\right\}$. We take $\mathcal{C}_{9}=\left\{a_{2}, b_{1}, b_{2}, d_{1}, d_{2}, g_{1}, g_{2}, h, v\right\}$ if $x=h$ or $\mathcal{C}_{9}=$ $\left.\overline{\left\{a_{2}, c_{1}, c_{2}\right.}, d_{1}, d_{2}, g_{1}, g_{2}, h^{\prime}, v\right\}$ if $x=h^{\prime}$. Thus, $\mathcal{C}_{9}$ is a vertex cover of $D$ with $L_{3}\left(\mathcal{C}_{9}\right)=\{x\}$. Also, $y \in N_{G}(x) \subseteq\left\{v, d_{1}, d_{2}, g_{1}, g_{2}\right\}$. If $y=v$, then $\{x, z\} \subseteq N_{D}(y)=\left\{h, h^{\prime}\right\}$. Hence, $N_{D}^{+}(y)=\{x\} \subseteq \mathcal{C}_{9}$, then $y \in \mathcal{C}_{9} \backslash L_{1}\left(\mathcal{C}_{9}\right)$. Now, if $y \neq v$, then $y \in\left\{d_{1}, d_{2}\right\}$ when $x=h$ or $y \in\left\{g_{1}, g_{2}\right\}$ when $x=h^{\prime}$. Then, $N_{D}(y) \backslash \mathcal{C}_{9} \subseteq\left\{a_{1}, c_{1}, c_{2}\right\} \subseteq V_{2}$ if $x=h$ or $N_{D}(y) \backslash \mathcal{C}_{9} \subseteq\left\{b_{1}, b_{2}\right\} \subseteq V_{2}$ if $x=h^{\prime}$. Consequently, by Lemma 5.7, $N_{D}(y) \backslash \mathcal{C}_{9} \subseteq N_{D}^{-}(y)$, since $y \in V^{+}$. Thus, $N_{D}^{+}(y) \subseteq \mathcal{C}_{9}$ and $y \in \mathcal{C}_{9} \backslash L_{1}\left(\mathcal{C}_{9}\right)$. Therefore, we take $\mathcal{C}=\overline{\mathcal{C}}_{9}$.

Theorem 5.10 Let $D=(G, \mathcal{O}, w)$ be a connected weighted oriented graph without 4and 5-cycles. Hence, $I(D)$ is unmixed if and only if $D$ satisfies one of the following conditions:

(a) $G \in\left\{C_{7}, T_{10}\right\}$ and the vertices of $V^{+}$are sinks.

(b) Each vertex is in exactly one simplex of $D$ and each simplex of $D$ has no generating

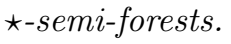

Proof. $\Rightarrow)$ By (3) in Theorem 2.12 and Remark 2.17, $G$ is well-covered. Thus, by Theorem 2.28, $G \in\left\{C_{7}, T_{10}\right\}$ or $\left\{V(H) \mid H \in S_{G}\right\}$ is a partition of $V(G)$. If $G \in\left\{C_{7}, T_{10}\right\}$, then by Proposition [5.9. $D$ satisfies (a). Now, if $\left\{V(H) \mid H \in S_{G}\right\}$ is a partition of $V(G)$, then $G$ is an $S C Q$ graph with $C_{G}=Q_{G}=\emptyset$. Hence, by Theorem 4.8, $D$ satisfies (b).

$\Leftarrow$ ) If $D$ satisfies (a), then by Theorem 2.28 , $G$ is well-covered. Consequently, by Corollary 5.2, $I(D)$ is unmixed. Now, if $D$ satisfies (b), then $G$ is an $S C Q$ graph, with $C_{G}=Q_{G}=$ $\emptyset$. Therefore, by (b) and Theorem 4.8, $I(D)$ is unmixed. 
Corollary 5.11 Let $D$ be a weighted oriented graph without isolated vertices and $\operatorname{girth}(G) \geqslant 6$. Hence, $I(D)$ is unmixed if and only if $D$ satisfies one of following properties:

(a) $G=C_{7}$ and the vertices of $V^{+}$are sinks.

(b) $G$ has a perfect matching $e_{1}=\left\{x_{1}, x_{1}^{\prime}\right\}, \ldots, e_{r}=\left\{x_{r}, x_{r}^{\prime}\right\}$ where $\operatorname{deg}_{D}\left(x_{1}^{\prime}\right)=\cdots=$ $\operatorname{deg}_{D}\left(x_{r}^{\prime}\right)=1$ and $\left(x_{j}^{\prime}, x_{j}\right) \in E(D)$ if $x_{j} \in V^{+}$.

Proof. $\Leftarrow$ ) If $D$ satisfies (a), then $I(D)$ is unmixed by (a) in Theorem [5.10 Now, assume $D$ satisfies (b). Assume $b^{\prime} \in N_{D}^{+}(a)$ with $a \in V^{+}$such that $\left\{b, b^{\prime}\right\}=e_{j}=$ $\left\{x_{j}, x_{j}^{\prime}\right\}$ for some $1 \leqslant j \leqslant r$. If $b^{\prime}=x_{j}^{\prime}$, then $x_{j}=a \in V^{+}$, since $\operatorname{deg}_{D}\left(x_{j}^{\prime}\right)=1$. So, $x_{j}^{\prime}=b^{\prime} \in N_{D}^{+}(a)=N_{D}^{+}\left(x_{j}\right)$. But by hypothesis, $\left(x_{j}^{\prime}, x_{j}\right) \in E(D)$, since $x_{j} \in V^{+}$. A contradiction, then $b^{\prime}=x_{j}$. Thus, $b=x_{j}^{\prime}$ implies $N_{D}(b)=\left\{x_{j}\right\}=\left\{b^{\prime}\right\} \subseteq N_{D}^{+}(a)$. Hence, by Proposition $4.1\left|\mathcal{C} \cap e_{j}\right|=1$ where $\mathcal{C}$ is a strong vertex cover. So, $|\mathcal{C}|=r$. Therefore, by (2) in Theorem 2.12, $I(D)$ is unmixed.

$\Rightarrow$ ) $G \neq T_{10}$, since $T_{10}$ has 3-cycles. Hence, by Theorem 5.10, $D$ satisfies (a) or $\{V(H) \mid$ $\left.H \in S_{G}\right\}$ is a partition of $V(G)$. Furthermore, $S_{G} \subseteq E(G)$, since $G$ has not isolated vertices and $\operatorname{girth}(G) \geqslant 6$. Thus, we can assume $S_{G}=\left\{e_{1}, \ldots, e_{r}\right\}$ where $e_{i}=\left\{x_{i}, x_{i}^{\prime}\right\}$ and $\operatorname{deg}_{D}\left(x_{i}^{\prime}\right)=1$ for $i=1, \ldots, s$. Also, by Theorem [5.10, each $e_{i}$ has no generating $\star$-semi-forests. So, by Theorem 3.10, $e_{i} \not \subset \mathcal{C}$ for each strong vertex cover $\mathcal{C}$. Consequently, $\left|e_{i} \cap \mathcal{C}\right|=1$, since $\mathcal{C}$ is a vertex cover. Then, $e_{i}$ satisfies (2) of Proposition 4.1. Now, suppose $\left(x_{j}, x_{j}^{\prime}\right) \in E(D)$ and $x_{j} \in V^{+}$. We take $a=b=x_{j}$ and $b^{\prime}=x_{j}^{\prime}$, then by (2) of Proposition 4.1, $N_{D}\left(x_{j}\right)=N_{D}(b) \subseteq N_{D}^{+}(a)=N_{D}^{+}\left(x_{j}\right)$. Hence, $x_{j}$ is a source. A contradiction, by Remark 2.3, since $x_{j} \in V^{+}$. Therefore, $D$ satisfies (b).

Proposition 5.12 If $G=P_{10}$, then the following properties are equivalent:

(1) $I(D)$ is unmixed.

(2) If $y \in V^{+}$and $y$ is not a sink, then $y=d_{1}$ with $N_{D}^{+}(y)=\left\{g_{1}, b_{2}\right\}$ or $y=d_{2}$ with $N_{D}^{+}(y)=\left\{g_{2}, b_{1}\right\}$.

Proof. $(2) \Rightarrow(1)$ Let $\mathcal{C}$ be a strong vertex cover of $D$. Suppose $x \in L_{3}(\mathcal{C})$. Then, there is $y \in\left(\mathcal{C} \backslash L_{1}(\mathcal{C})\right) \cap V^{+}$such that $x \in N_{D}^{+}(y)$. Thus, by (2), $y \in\left\{d_{1}, d_{2}\right\}$ and $x \in N_{D}^{+}(y) \subseteq\left\{b_{1}, b_{2}, g_{1}, g_{2}\right\}$. Hence, $L_{3}(\mathcal{C}) \subseteq\left\{b_{1}, b_{2}, g_{1}, g_{2}\right\}$. By symmetry of $P_{10}$, we can assume $y=d_{1}$. Then by (2), $x \in N_{D}^{+}(y)=\left\{g_{1}, b_{2}\right\}$. Also, $\left\{g_{1}, d_{1}, b_{2}\right\}=N_{D}^{+}[y] \subseteq \mathcal{C}$, since $y \in \mathcal{C} \backslash L_{1}(\mathcal{C})$. But $y=d_{1} \notin L_{3}(\mathcal{C})$, then $N_{D}(y) \nsubseteq \mathcal{C}$. Thus, $d_{2} \notin \mathcal{C}$. So, by Remark 2.8. $\left\{b_{1}, d_{1}, g_{2}\right\}=N_{D}\left(d_{2}\right) \subseteq \mathcal{C}$. Furthermore, $\left\{a_{1}, a_{2}\right\} \cap L_{3}(\mathcal{C})=\emptyset$, since $L_{3}(\mathcal{C}) \subseteq$ $\left\{b_{1}, b_{2}, g_{1}, g_{2}\right\}$. Consequently, $\left\{a_{1}, a_{2}\right\} \cap \mathcal{C}=\emptyset$, since $N_{D}\left(a_{1}, a_{2}\right)=\left\{g_{1}, b_{1}, g_{2}, b_{2}\right\} \subseteq$ $\mathcal{C}$. But, $x \in\left\{g_{1}, b_{2}\right\} \cap L_{3}(\mathcal{C})$, then $a_{1} \in N_{D}\left(g_{1}\right) \subseteq \mathcal{C}$ or $a_{2} \in N_{D}\left(b_{2}\right) \subseteq \mathcal{C}$. Hence, $\left\{a_{1}, a_{2}\right\} \cap \mathcal{C} \neq \emptyset$. A contradiction, then $L_{3}(\mathcal{C})=\emptyset$. Also, by Theorem 2.28 and Remark 2.17, $I(G)$ is unmixed. Therefore, by (3) in Theorem 2.12, $I(D)$ is unmixed.

$(1) \Rightarrow(2)$ We take $V_{2}:=\left\{a \in V(G) \mid \operatorname{deg}_{G}(a)=2\right\}$ and $y \in V^{+}$, such that $y$ is not a sink, then there is $(y, x) \in E(D)$. Also, by Remark 2.3, there is $z \in N_{D}^{-}(y)$. We will prove $y \in\left\{d_{1}, d_{2}\right\}$. If $\operatorname{deg}_{D}(x)=2$, then by Lemma 5.7, $y \in\left\{d_{1}, d_{2}\right\}$. Now, we assume $\operatorname{deg}_{D}(x) \geqslant 3$, then by symmetry of $P_{10}$, we can suppose $x \in\left\{g_{1}, d_{1}\right\}$.

First suppose $x=g_{1}$. Thus, $y \in N_{D}\left(g_{1}\right)=\left\{a_{1}, c_{1}, d_{1}\right\}$. If $y \in\left\{a_{1}, c_{1}\right\}$, then $\operatorname{deg}_{D}(y)=2$ and $y \in \mathcal{C}_{1}=\left\{a_{1}, a_{2}, c_{1}, d_{1}, d_{2}, g_{1}, g_{2}\right\}$ is a vertex cover of $D$ with $L_{3}\left(\mathcal{C}_{1}\right)=\{x\}$. Hence, 
$N_{D}(y)=\{x, z\}$ implies $N_{D}^{+}(y)=\{x\}=\left\{g_{1}\right\} \subseteq \mathcal{C}_{1}$. Consequently, $y \in \mathcal{C}_{1} \backslash L_{1}\left(\mathcal{C}_{1}\right)$. Hence, $\mathcal{C}_{1}$ is strong, since $L_{3}\left(\mathcal{C}_{1}\right)=\{x\}$. A contradiction, by Theorem 2.12, then $y=d_{1}$. Now, suppose $x=d_{1}$. Then, $\mathcal{C}_{2}=\left\{a_{1}, b_{2}, c_{2}, d_{1}, d_{2}, g_{1}, g_{2}\right\}$ is a vertex cover of $D$ with $L_{3}\left(\mathcal{C}_{2}\right)=\{x\}$. Also, $y \in N_{D}(x)=\left\{g_{1}, b_{2}, d_{2}\right\}$. If $y \in\left\{g_{1}, b_{2}\right\}$, then $N_{D}(y) \backslash\left\{d_{1}\right\} \subseteq$ $N_{D}\left(g_{1}, b_{2}\right) \backslash\left\{d_{1}\right\}=\left\{a_{1}, a_{2}, c_{1}\right\} \subseteq V_{2}$. Hence, by Lemma 5.7, $N_{D}(y) \backslash\left\{d_{1}\right\} \subseteq N_{D}^{-}(y)$, since $y \notin\left\{b_{1}, b_{2}\right\}$. Thus, $N_{D}^{+}(y)=\left\{d_{1}\right\}=\{x\} \subseteq \mathcal{C}_{2}$ implying $y \in \mathcal{C}_{2} \backslash L_{1}\left(\mathcal{C}_{2}\right)$. Consequently, $\mathcal{C}_{2}$ is strong with $L_{3}\left(\mathcal{C}_{2}\right) \neq \emptyset$. A contradiction, by Theorem 2.12, then $y=d_{2}$.

Therefore $y \in\left\{d_{1}, d_{2}\right\}$. By symmetry of $P_{10}$, we can assume $y=d_{1}$. Now, we will prove $N_{D}^{+}(y)=\left\{g_{1}, b_{2}\right\}$. By contradiction, in each one of the following cases, we give a strong vertex cover $\mathcal{C}^{\prime}$ with $L_{3}\left(\mathcal{C}^{\prime}\right) \neq \emptyset$, since $I(D)$ is unmixed and $z \in N_{D}^{-}(y)$.

Case (1) $g_{1} \notin N_{D}^{+}\left(d_{1}\right)$ and $b_{2} \in N_{D}^{+}\left(d_{1}\right)$. So, $N_{D}^{+}\left(d_{1}\right) \subseteq\left\{b_{2}, d_{2}\right\}$ and $\mathcal{C}_{1}^{\prime}=$ $\left\{a_{1}, a_{2}, b_{2}, c_{1}, c_{2}, d_{1}, d_{2}\right\}$ is a vertex cover of $D$ with $L_{3}\left(\mathcal{C}_{1}^{\prime}\right)=\left\{b_{2}\right\}$. Also, $\left(d_{1}, b_{2}\right) \in E(D)$ and $y=d_{1} \in\left(\mathcal{C}_{1}^{\prime} \backslash L_{1}\left(\mathcal{C}_{1}^{\prime}\right)\right) \cap V^{+}$, since $b_{2} \in N_{D}^{+}\left[d_{1}\right] \subseteq\left\{d_{1}, b_{2}, d_{2}\right\} \subset \mathcal{C}_{1}^{\prime}$. Hence, $\mathcal{C}_{1}^{\prime}$ is a strong vertex cover of $D$.

Case (2) $b_{2} \notin N_{D}^{+}\left(d_{1}\right)$ and $g_{1} \in N_{D}^{+}\left(d_{1}\right)$. Then, $N_{D}^{+}\left(d_{1}\right) \subseteq\left\{g_{1}, d_{2}\right\}$ and $\mathcal{C}_{2}^{\prime}=$ $\left\{a_{1}, a_{2}, c_{1}, d_{1}, d_{2}, g_{1}, g_{2}\right\}$ is a vertex cover of $D$ with $L_{3}\left(\mathcal{C}_{2}^{\prime}\right)=\left\{g_{1}\right\}$. Furthermore $\left(d_{1}, g_{1}\right) \in$ $E(D)$ and $d_{1}=y \in\left(\mathcal{C}_{2}^{\prime} \backslash L_{1}\left(\mathcal{C}_{2}^{\prime}\right)\right) \cap V^{+}$, since $g_{1} \in N_{D}^{+}\left[d_{1}\right] \subseteq\left\{d_{1}, g_{1}, d_{2}\right\} \subset \mathcal{C}_{2}^{\prime}$. Hence, $\mathcal{C}_{2}^{\prime}$ is a strong vertex cover of $D$.

Case (3) $b_{2}, g_{1} \notin N_{D}^{+}\left(d_{1}\right)$. Thus, $z=d_{1}, N_{D}^{+}\left(d_{1}\right)=\left\{d_{2}\right\}$ and $\mathcal{C}_{3}^{\prime}=$ $\left\{a_{2}, b_{1}, c_{1}, d_{1}, d_{2}, g_{1}, g_{2}\right\}$ is a vertex cover of $D$ with $L_{6}\left(\mathcal{C}_{3}^{\prime}\right)=\left\{d_{2}\right\}$. Also, $\left(d_{1}, d_{2}\right) \in E(D)$ and $d_{1}=y \in\left(\mathcal{C}_{3}^{\prime} \backslash L_{1}\left(\mathcal{C}_{3}^{\prime}\right)\right) \cap V^{+}$, since $N_{D}^{+}\left[d_{1}\right]=\left\{d_{1}, d_{2}\right\} \subset \mathcal{C}_{3}^{\prime}$. Hence, $\mathcal{C}_{3}^{\prime}$ is a strong vertex cover of $D$.

Theorem 5.13 Let $D=(G, \mathcal{O}, w)$ be a connected weighted oriented graph, with $\operatorname{girth}(G) \geqslant 5$. Hence, $I(D)$ is unmixed if and only if $D$ satisfies one of the following properties:

(a) $G \in\left\{K_{1}, C_{7}, Q_{13}, P_{13}, P_{14}\right\}$ and the vertices of $V^{+}$are sinks.

(b) $G=P_{10}$, furthermore if $y$ is not a sink in $V^{+}$, then $y=d_{1}$ with $N_{D}^{+}(y)=\left\{g_{1}, b_{2}\right\}$ or $y=d_{2}$ with $N_{D}^{+}(y)=\left\{g_{2}, b_{1}\right\}$.

(c) Each vertex is in exactly one simplex of $G$ or in exactly one basic 5-cycle of $G$. Furthermore, each simplex of $D$ has not a generating $\star$-semi-forest and each basic 5 -cycle of $D$ has the $\star$-property.

Proof. $\Rightarrow)$ By $(3)$ in Theorem 2.12 and Remark 2.17 $G$ is well-covered. By Theorem 2.30. $G \in\left\{K_{1}, C_{7}, P_{10}, P_{13}, P_{14}, Q_{13}\right\}$ or $\left\{V(H) \mid H \in S_{G} \cup C_{G}\right\}$ is a partition of $V(G)$. If $\left\{V(H) \mid H \in S_{G} \cup C_{G}\right\}$ is a partition of $V(G)$, then $G$ is an $S C Q$ graph with $Q_{G}=\emptyset$. Hence, by Theorem 4.8, $D$ satisfies (c). Now, if $G=P_{10}$, then by Proposition 5.12, $D$ satisfies (b). Furthermore, if $G \in\left\{C_{7}, Q_{13}, P_{13}, P_{14}\right\}$, then by Proposition 5.9, $D$ satisfies (a). Finally, if $G=K_{1}$, then by Remark $2.3, V^{+}=\emptyset$.

$\Leftarrow$ ) If $D$ satisfies (b), then by Proposition 5.12 $I(D)$ is unmixed. Now, if $D$ satisfies (c), then $G$ is an $S C Q$ graph with $Q_{G}=\emptyset$. Consequently, by Theorem 4.8, $I(D)$ is unmixed. Finally, if $D$ satisfies (a), then by Theorem 2.30, $G$ is well-covered. Therefore, by Corollary 5.2, $I(D)$ is unmixed.

Remark 5.14 A graph is well-covered if and only if each connected component is wellcovered. Hence, when $D$ is no connected in Theorem 5.10 (resp. 5.13), $I(D)$ is unmixed if and only if each connected component of $D$ satisfies (a) or (b) (resp. (a), (b) or (c)). 


\section{$6 \quad$ Examples}

Example 6.1 Let $D_{1}$ be the following weighted oriented graph.

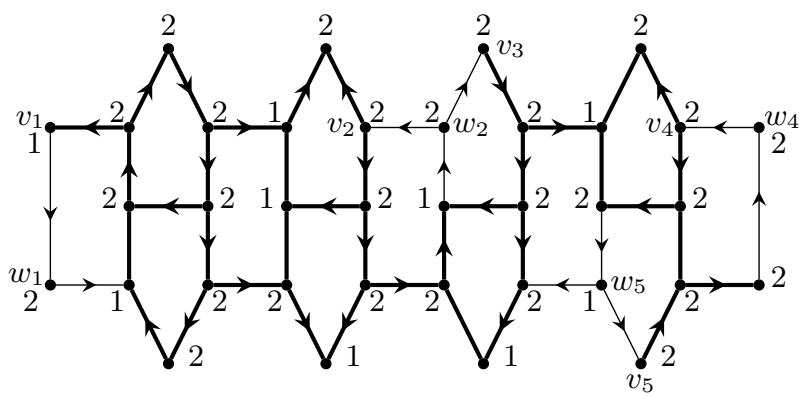

We take the weighted oriented subgraph $K$ of $D_{1}$ induced by $V\left(D_{1}\right) \backslash\left\{w_{1}, w_{2}, w_{4}, w_{5}\right\}$. In the following figure, $T_{1}, \ldots, T_{5}$ are the ROT's and $B_{1}$ is the unicycle oriented subgraph such that the parent of $T_{i}$ is $v_{i}$ and $W^{T_{i}}=\left\{w_{i}\right\}$ for $i=1, \ldots, 5$.

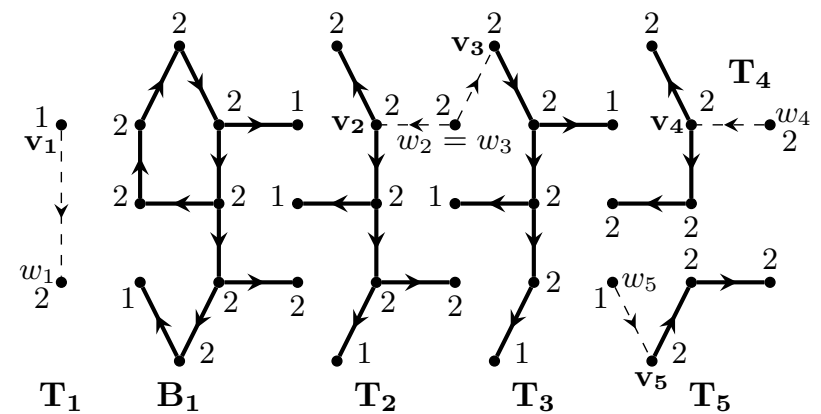
Furthermore, $H=\cup_{i=1}^{5} T_{i} \cup B_{1}$ is a $\star$-semi-forest with $W_{1}^{H}=$ $\left\{w_{1}, w_{5}\right\} W_{2}^{H}=\left\{w_{2}, w_{4}\right\}$ and $V(H)=V(K)$. Therefore, $H$ is a generating $\star$-semi-forest of $K$.

Example 6.2 Let $D_{2}$ be an oriented weighted graph such as the Figure. Let $K^{4}=H=$ $D_{2}\left[x_{1}, x_{2}, x_{3}\right]$ be an induced weighted oriented subgraph of $D_{2}$ and $H$ a generating $\star$-semiforest of $K^{4}$ with $\tilde{H}=H=\left\{x_{1}, x_{2}, x_{3}\right\} \subseteq V^{+}$and $W_{1}=W_{2}=\emptyset$. Then, by Theorem 3.10, there is a strong vertex cover $\mathcal{C}$ of $D_{2}$, such that $V\left(K^{4}\right) \subseteq \mathcal{C}$. For this purpose, first we take a minimal vertex cover $\mathcal{C}_{i}$ of $D$ and define $\mathcal{C}_{i}^{\prime}=\left(\mathcal{C}_{i} \backslash W_{1}\right) \cup N_{D}\left(W_{1}\right) \cup N_{D}^{+}\left(W_{2} \cup \tilde{H}\right)$. Finally, we use the algorithm in the proof of the Proposition 3.1.

In this example, $\mathcal{C}_{i}^{\prime}=\mathcal{C}_{i} \cup N_{D}^{+}(\tilde{H})=\mathcal{C}_{i} \cup N_{D}^{+}\left(\left\{x_{1}, x_{2}, x_{3}\right\}\right)=\mathcal{C}_{i} \cup\left\{x_{1}, x_{2}, x_{3}, y_{1}, y_{2}\right\}$.

- If we take $\mathcal{C}_{1}=\left\{x_{1}, x_{2}, y_{1}, y_{2}, y_{3}\right\}$ as a minimal vertex cover of $D_{2}$, then $\mathcal{C}_{1}^{\prime}=\left\{x_{1}, x_{2}, x_{3}, y_{1}, y_{2}, y_{3}\right\}$

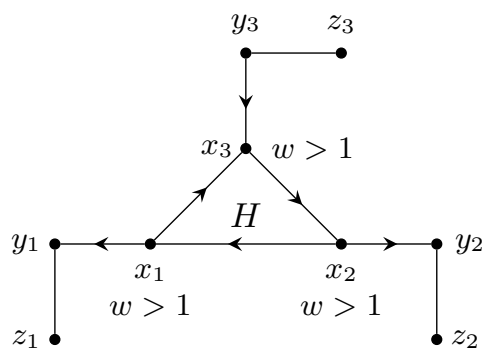
and $L_{3}\left(\mathcal{C}_{1}^{\prime}\right) \backslash N_{D}^{+}(\tilde{H})=\emptyset$. Thus, by Proposition 3.1 $\mathcal{C}=\mathcal{C}_{1}^{\prime}=\left\{x_{1}, x_{2}, x_{3}, y_{1}, y_{2}, y_{3}\right\}$ is a strong vertex cover such that $V\left(K^{4}\right) \subseteq \mathcal{C}$ and it is no minimal. Furthermore, in this case it is enough to know the orientation of $E\left(K^{4}\right)$, since $L_{3}(\mathcal{C})=\left\{x_{1}, x_{2}, x_{3}\right\}$ and $N_{D_{2}}\left(x_{i}\right) \subseteq \mathcal{C}$ for $i=1,2,3$.

- Now, if we take $\mathcal{C}_{2}=\left\{x_{1}, x_{2}, x_{3}, z_{1}, z_{2}, z_{3}\right\}$ as a minimal vertex cover of $D_{2}$, then $\mathcal{C}_{2}^{\prime}=V\left(D_{2}\right) \backslash$ $\left\{y_{3}\right\}$ and $L_{3}\left(\mathcal{C}_{2}^{\prime}\right) \backslash N_{D}^{+}(\tilde{H})=\left\{z_{1}, z_{2}\right\}$. Thus, by the algorithm in the proof of Proposition 3.1, $\mathcal{C}^{\prime}=$ $\mathcal{C}_{4}^{\prime}=\left\{x_{1}, x_{2}, x_{3}, y_{1}, y_{2}, z_{3}\right\}$ is a strong vertex cover such that $V\left(K^{4}\right) \subseteq \mathcal{C}^{\prime}$. 
Since $\mathcal{C}$ and $\mathcal{C}^{\prime}$ are no minimal with $L_{3}(\mathcal{C})=\left\{x_{1}, x_{2}, x_{3}\right\}$ and $L_{3}\left(\mathcal{C}^{\prime}\right)=\left\{x_{1}, x_{2}\right\}$, then $D_{2}$ is mixed. Furthermore, $V\left(D_{2}\right)$ has a partition in complete graphs: $K^{i}=D_{2}\left[y_{i}, z_{i}\right]$ for $i=1,2,3$ and $K^{4}=D_{2}\left[x_{1}, x_{2}, x_{3}\right]$.

Example 6.3 Let $D_{3}=(G, \mathcal{O}, w)$ be the following oriented graph. Hence,

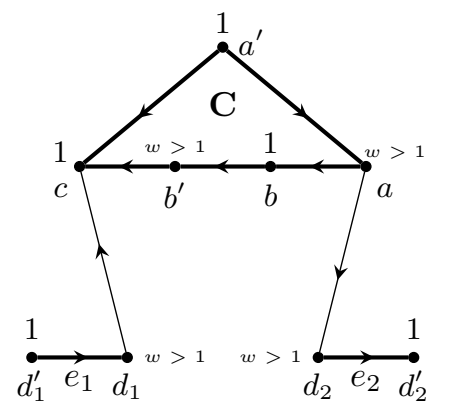

- $G$ has no 3- and 4-cycles. Moreover, $\operatorname{girth}(G)=5$.

- $G$ is an $S C Q$-graph with $S_{G}=\left\{e_{1}, e_{2}\right\}, C_{G}=\{C\}$ and $Q_{G}=\emptyset$, where $e_{1}=\left\{d_{1}, d_{1}^{\prime}\right\}, e_{2}=\left\{d_{2}, d_{2}^{\prime}\right\}$ and $C=\left(a^{\prime}, a, b, b^{\prime}, c, a^{\prime}\right)$.

- $C$ has the $\star$-property. But $e_{2}$ has a generating $\star$-semi-forest. Hence, by Theorem [5.13, $I(D)$ is mixed.

Example 6.4 Let $D_{4}=(G, \mathcal{O}, w)$ be the following oriented weighted graph. Hence,

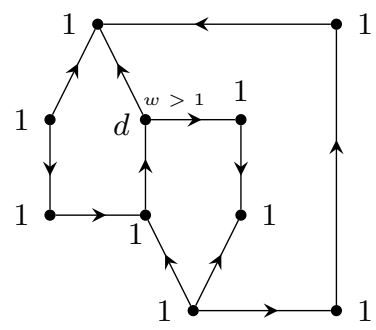

- $G=P_{10}$, then by Theorem 2.30 and Remark 2.17, $G$ is well-covered and $I(G)$ is unmixed.

- $d$ is not a sink and $d \in V^{+}$.

- By Proposition 5.12 $I\left(D_{4}\right)$ is unmixed.

\section{References}

[1] C. Carvalho, V. G. Neumann and H. H. López. Projective nested cartesian codes. Bull. Braz. Math. Soc. (N.S.), 48, (2017), no. 2, 283-302.

[2] I. D. Castrillón, R. Cruz, and E. Reyes. On well-covered, vertex decomposable and Cohen-Macaulay graphs. Electron. J. Combin., 23, (2016), no. 2, Paper 2.39, 17pp.

[3] I. Castrillón and E. Reyes, Pure vertex decomposable simplicial complex associated to graphs whose 5-cycles are chorded, Bol. Soc. Mat. Mex. 23 (2017), 399-412.

[4] R. Diestel, Graph Theory, Second Edition, Graduate Texts in Mathematics, 173, Springer-Verlag, New York, 2000.

[5] A. Finbow, B. Hartnell and R. J. Nowakowski, A characterization of well-covered graphs of girth 5 or greater, J. of Combinatorial Theory B 57 (1993) 44-68.

[6] A. Finbow, B. Hartnell and R. J. Nowakowski, A characterization of well-covered graphs that contain neither 4- nor 5-cycles, J. Graph Theory 18 (1994) 713-721. 
[7] P. Gimenez, J. Martínez-Bernal, A. Simis, R. H. Villarreal, and C. E. Vivares, Symbolic powers of monomial ideals and Cohen-Macaulay vertex-weighted digraphs, Singularities, Algebraic Geometry, Commutative Algebra, and Related Topics, Springer, Cham, (2018), 491-510.

[8] H. T. Há, K. N. Lin, S. Morey, E. Reyes and R. H. Villarreal, Edge ideals of oriented graphs, Internat. J. Algebra Comput., 29 (2019), no. 3, 535-559.

[9] J. Martínez-Bernal, Y. Pitones and R. H. Villarreal, Minimum distance functions of graded ideals and Reed-Muller-type codes. J. Pure Appl. Algebra 221 (2017), no. 2, $251-275$.

[10] Y. Pitones, E. Reyes and J. Toledo, Monomial ideals of weighted oriented graphs, Electronic. J. Combin., 26 (2019), no. 3, Paper 3.44, 18 pp.

[11] Y. Pitones, E. Reyes and R. H. Villarreal, Unmixed and Cohen-Macaulay weighted oriented König graphs, (2019), preprint, arXiv:1909.13295.

[12] E. Prisner, J. Topp and P. D. Vestergaard, Well Covered Simplicial, Chordal, and Circular Arc Graphs, J. Graph Theory, 21 (1996) 113-119.

[13] B. Randerath and L. Volkmann, A characterization of well-covered block-cactus graphs, Australas. J. Combin, 9 (1994) 307-314.

[14] R. H. Villarreal, Monomial Algebras, Second Edition, Monographs ans Research Notes in Mathematics, Chapman and Hall/CRC, 2015.

[15] G. Zhu, L. Xu, H. Wang and Z. Tang, Projective dimensions and regularity of edge ideal of some weighted oriented graphs, Rocky Mountain J. Math., 49 (2019), no. 4, 1391--1406. 